\title{
Discovery of a carbonic anhydrase-Rubisco supercomplex within the alpha-carboxysome
}

\author{
Cecilia Blikstad ${ }^{1,4}$, Eli J. Dugan ${ }^{1,5}$, Thomas G. Laughlin ${ }^{1,6}$, Mira D. Liu², Sophie R. Shoemaker ${ }^{1}$,
} Jonathan P. Remis ${ }^{3}$ and David F. Savage ${ }^{1}$

\author{
Affiliations: \\ ${ }^{1}$ Department of Molecular and Cell Biology, University of California, Berkeley, California 94720, \\ United States \\ ${ }^{2}$ Department of Chemistry, University of California, Berkeley, California 94720, United States \\ ${ }^{3}$ California Institute for Quantitative Biosciences (QB3), University of California, Berkeley, \\ California 94720, USA \\ ${ }^{4}$ Current affiliation: Department of Chemistry - Ångström Laboratory, Uppsala University, 75120 \\ Uppsala, Sweden \\ ${ }^{5}$ Current Affiliation: Tetrad Graduate Program, University of California at San Francisco, San \\ Francisco, CA, 94158 USA \\ ${ }^{6}$ Current affiliation: Division of Biological Sciences, University of California, San Diego, La Jolla, \\ CA 92093, USA
}

\section{ORCID:}

CB: 0000-0001-5740-926X

EJD: 0000-0003-2400-5511

TGL: 0000-0001-8943-1330

MDL: 0000-0002-4412-7770

SRS: 0000-0002-5278-2361

JPR: 0000-0001-8317-4513

DFS: 0000-0003-0042-2257 


\section{ABSTRACT}

Carboxysomes are proteinaceous organelles that encapsulate key enzymes of $\mathrm{CO}_{2}$ fixation, Rubisco and carbonic anhydrase, and are the centerpiece of the bacterial $\mathrm{CO}_{2}$ concentrating mechanism (CCM). In the CCM, actively accumulated cytosolic bicarbonate diffuses into the carboxysome and is converted to $\mathrm{CO}_{2}$ by carbonic anhydrase, producing a high $\mathrm{CO}_{2}$ concentration near Rubisco and ensuring efficient carboxylation. Self-assembly of the a-carboxysome is orchestrated by the intrinsically disordered scaffolding protein, CsoS2, which interacts with both Rubisco and carboxysomal shell proteins, but it is unknown how CsoSCA, the carbonic anhydrase, is incorporated into the a-carboxysome. Here, we present the structural basis of carbonic anhydrase encapsulation into a-carboxysomes from Halothiobacillus neapolitanus. We find that CsoSCA interacts directly with Rubisco via an intrinsically disordered $\mathrm{N}$-terminal domain. A $1.98 \AA$ single-particle cryo-electron microscopy structure of Rubisco in complex with this peptide reveals that CsoSCA binding is predominantly mediated by a network of hydrogen bonds. CsoSCAs binding site overlaps with that of CsoS2 but the two proteins utilize substantially different motifs and modes of binding, revealing a plasticity of the Rubisco binding site. Our results advance the understanding of biogenesis of carboxysomes and highlights the importance of Rubisco, not only as an enzyme, but also as a hub protein central for assembling supercomplexes. 


\section{Introduction}

Carbonic anhydrase (CA) catalyses the rapid interconversion between carbon dioxide $\left(\mathrm{CO}_{2}\right)$ and bicarbonate $\left(\mathrm{HCO}_{3}^{-}\right)$. Due to the centrality of this reaction in metabolism, $\mathrm{CA}$ is an essential gene in all organisms where it has been tested. In photosynthesis, CA's role is often to supply the enzyme Rubisco - the carboxylase of the CBB cycle - with $\mathrm{CO}_{2}$ to ensure fast fixation (1). Rubisco has modest turnover numbers and fails to distinguish between $\mathrm{CO}_{2}$ and the competing off-target substrate of $\mathrm{O}_{2}$ (2-4). To overcome Rubisco's limitation, plants, algae and bacteria have evolved different types of $\mathrm{CO}_{2}$ Concentrating Mechanisms (CCMs) which concentrate $\mathrm{CO}_{2}$ near Rubisco (5). This ensures saturation of Rubisco's active sites with $\mathrm{CO}_{2}$, competitive inhibition of oxygenation, and an increase in overall carbon assimilation rates. Importantly, to understand the role of a CA in a CCM, it is essential to understand enzyme localization and regulation (1).

The bacterial CCM is present in all Cyanobacteria and many Proteobacteria and consists of two main components: (I) energy-coupled inorganic carbon transporters that actively accumulate bicarbonate in the cytosol and (II) a proteinaceous bacterial organelle called the carboxysome, which co-encapsulates Rubisco and CA within a capsid-like protein shell (6-9). The accumulated $\mathrm{HCO}_{3}^{-}$diffuses into the carboxysome where it is rapidly converted to $\mathrm{CO}_{2}$ by $\mathrm{CA}$ while diffusion out of the structure is likely restricted by the shell $(10,11)$. This produces a locally high $\mathrm{CO}_{2}$ concentration within the carboxysome and enables efficient Rubisco carboxylation (12).

Microbiology and biochemistry shows there are, in fact, two types of carboxysomes which have evolved convergently $(6,13)$. These are the a-type found in oceanic Cyanobacteria and Proteobacteria and the $\beta$-type found in freshwater Cyanobacteria. In both instances, CA localization in the carboxysome is essential for growth in present day atmospheric $\mathrm{CO}_{2}$ concentrations (14-16). In contrast, CA activity in the cytosol has been shown to short-circuit the CCM leading to high $\mathrm{CO}_{2}$ requiring strains (17). Efficient encapsulation and regulation of CA activity is thus crucial for cell survival. All $\alpha$-carboxysomes contain a $\beta-C A$, CsoSCA (18-21), while $\beta$-carboxysomes have either an active $\gamma$-CA domain on the scaffolding protein $\mathrm{CcmM}(22$, 23) or a $\beta-C A$ named CcaA (24). While the mechanism of CA incorporation into the $\beta$-carboxysome is understood (25), it is unknown how CsoSCA incorporates into the a-carboxysome.

CsoSCA belongs to its own subclass of $\beta$-CAs and uniquely consists of three domains: an $\mathrm{N}$-terminal domain, a middle/catalytic domain and a C-terminal domain (Fig. 1B) (20). X-ray structural analysis has shown that the catalytic domain contains the zinc binding site as well as catalytic residues essential for CA activity. The C-terminal domain appears to be an ancient gene duplication of the catalytic domain but lacks the zinc binding residues. The $\mathrm{N}$-terminal domain (NTD) consists of an unstructured $\mathrm{N}$-terminal peptide followed by a $\sim 100$ residue 
a-helical domain, which lacks homology to any other known protein. The function of this domain is mysterious and has been speculated to be involved in the encapsulation process (20).

Here, we used biolayer interferometry (BLI) to screen CsoSCA for binding to all a-carboxysome proteins and identified Rubisco as its interaction partner. We show that the Rubisco interaction and encapsulation into carboxysomes is dependent on CsoSCA's unique intrinsically disordered $\mathrm{N}$-terminal peptide. Using this peptide, we targeted foreign cargo into the carboxysome, demonstrating that this sequence is sufficient for encapsulation. We further determined a $1.98 \AA$ single-particle cryo-electron microscopy (cryo-EM) structure of Rubisco in complex with the NTD peptide. The structure reveals that the peptide interacts with Rubisco at a site overlapping with a recently identified site responsible for targeting Rubisco to the a-carboxysome via interaction with the carboxysomal scaffolding protein CsoS2 (26). Thus, our work identifies a previously unknown supercomplex found inside the a-carboxysome and highlights a surprising flexibility in the scope of protein-protein interactions which lead to a-carboxysome self-assembly.

\section{Results}

\section{CsoSCA's N-terminus is necessary and sufficient for encapsulation}

In order to identify putative mechanisms for encapsulation of CsoSCA into the carboxysome, we first started with a bioinformatic examination of the CsoSCA protein. Phylogenetic analysis revealed that CsoSCA from cyanobacteria and proteobacteria cluster into two separate subfamilies (Fig. 1A and SI Fig 1). The cyanobacterial subfamily divides into two clusters. The proteobacteria subfamily is more diverse but has three distinct clusters, including a transition cluster more closely related to the cyanobacterial subfamily. Multiple sequence alignment (MSA) and calculated conservation score reveal a poorly conserved N-terminal region of the NTD while the rest of the protein is highly conserved (Fig. 1C). In our model organism, the a-proteobacteria $H$. neapolitanus, the sequence conservation of CsoSCA starts with residue H51. Using representatives from the different clusters of the phylogenetic tree, it was revealed that this 30-130 amino acid long NTD is predicted to be disordered (Fig 1C). Even though the NTD primary sequence is not conserved, its existence among all species is suggestive of a function.

To investigate the role of CsoSCA's unstructured NTD-peptide, a csoSCA deletion strain of the native organism $H$. neapolitanus $(\triangle c s o S C A$ ) was complemented with a version of CsoSCA lacking its first 48 residues $(\triangle 48 c s o S C A)$. The $\triangle 48 c s o S C A$ strain failed to grow in air (Fig. 1E) whereas complementation with full-length CsoSCA rescued growth, indicating an essential role for CsosCA's NTD. Synthetic carboxysome operons containing truncated CsoSCAs ( $\triangle 36 c s o S C A$ and $\triangle 48 c s o S C A$ ) were heterologously expressed in E. coli. Western blot analysis of enriched carboxysome (Fig. 1D) fractions showed that neither of these constructs produced carboxysomes containing CsoSCA, suggesting that the growth defect seen in the phenotyping experiment is due to an inability to encapsulate CsoSCA into the carboxysome when the $\mathrm{N}$-terminal peptide (N48-peptide) is removed. 
A

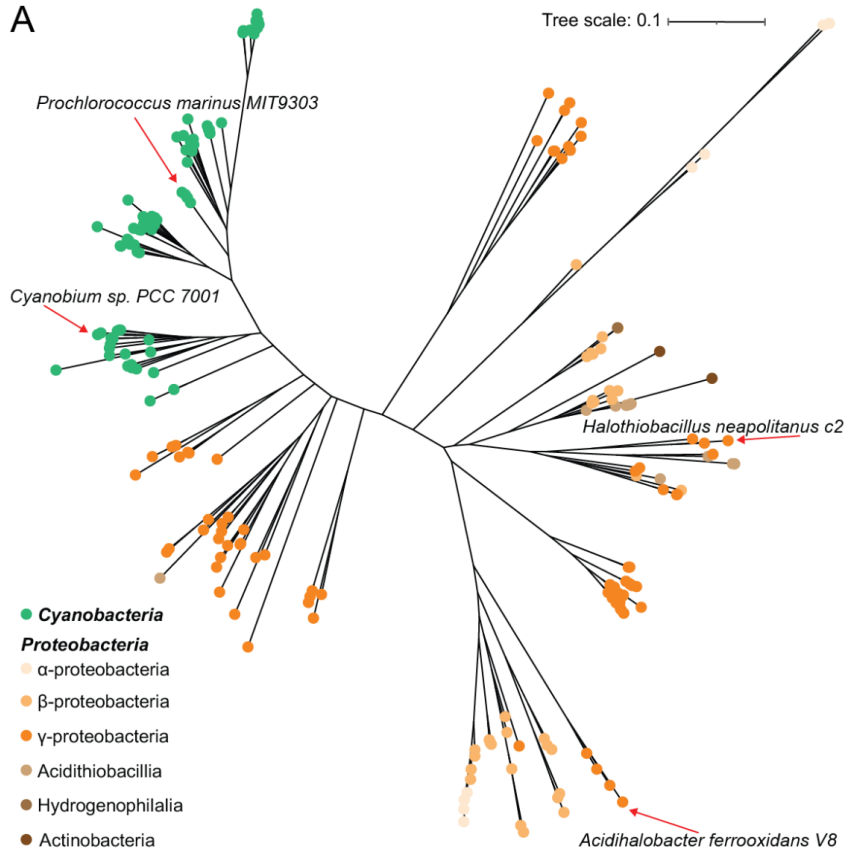

D

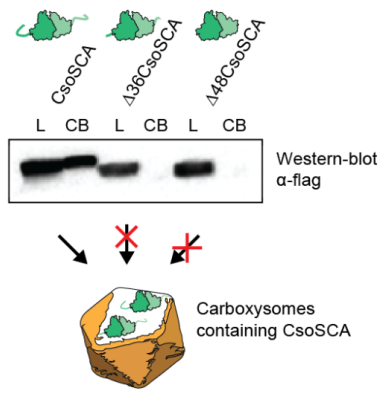

E

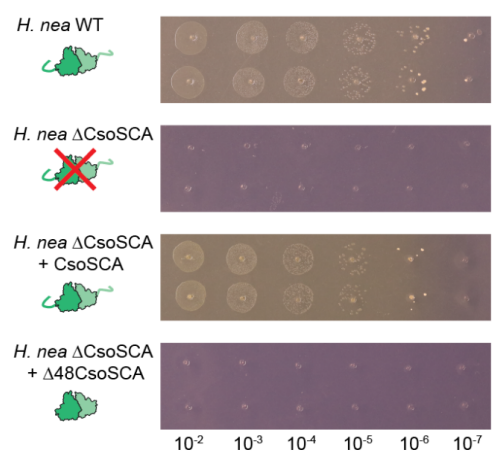

B

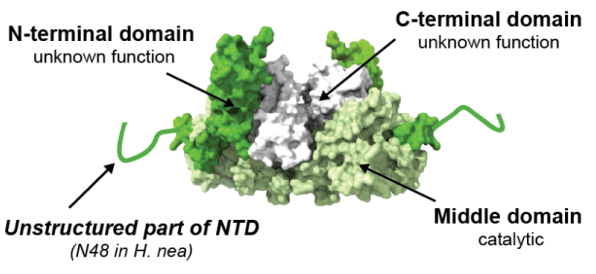

C

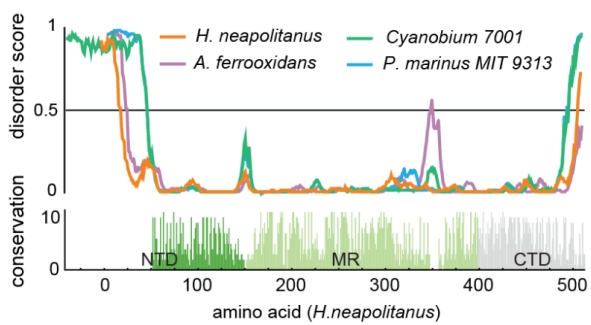

$\mathrm{F}$
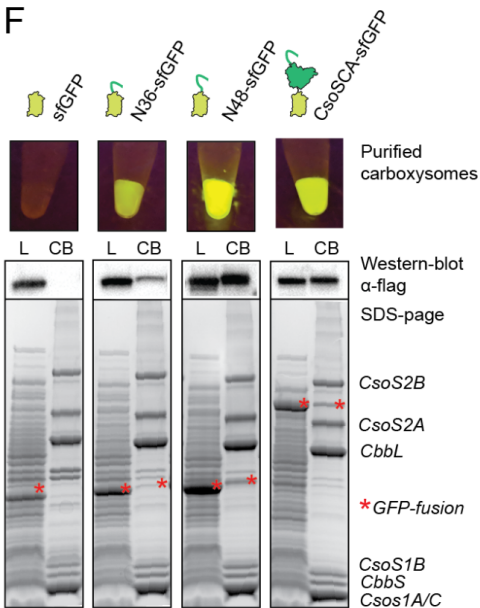

Figure 1: An intrinsically disordered, poorly conserved $\mathbf{N}$-terminal peptide is essential and sufficient for CsoSCA encapsulation.

(A) Maximum-likelihood phylogenetic tree of CsoSCA. Cyanobacterial homologs are colored in green and proteobacteria homologous in an orange/brown gradient. Scale bar, 0.1 substitutions per site. (B) Surface represented structure of CsoSCA dimer from H.neapolitanus (pdb: $2 \mathrm{fgy}$ ). $\mathrm{N}$-terminal domain (dark green) consists of a $\sim 50$ aa long unstructured peptide followed by a folded alpha-helical domain with unknown function. Middle domain (light green) contains the active site. The C-terminal domain (white) appears to be a gene duplication of the catalytic domain but lacks essential active site residues. (C) Disorder score of four representative CsoSCA homologous calculated using Disopred3 and conservation calculated from multiple sequence alignment. (D) Western-blot analysis of enriched carboxysomes produced heterologously in E. coli. Expressed carboxysome operons contained wild-type CsoSCA or $\mathrm{N}$-terminal truncated variants $\triangle 36 \mathrm{csoSCA}$ and $\triangle 48 \mathrm{csoSCA}$. Western-blot showing lysate (L) and enriched carboxysomes (CB) fractions detecting C-terminally flag-tagged CsoSCA variants. CsoSCA is not detected in the carboxysome fraction when $\mathrm{N}$-terminally truncated. (E) Complementation of full-length CsoSCA rescues growth of a CsoSCA knock-out in H.neapolitanus while complementation with a NTD truncated variant, d48CsoSCA, fails to rescue growth. (F) Fusing the unstructured NTD of CsoSCA (36 or 48 residues) to sfGFP targets the fusion protein to carboxysomes produced heterologously in E. coli while untagged sfGFP do not target to carboxysomes. The control with full-length CsoSCA-sfGFP also produces fluorescent carboxysomes. Panel shows fluorescence of purified carboxysomes, western-blot analysis against flag-tagged sfGFP and SDS-PAGE of purified carboxysomes. 
To further test the role of the CsoSCA-NTD in encapsulation, we sought to target foreign cargo into the carboxysome via fusion with peptides derived from the NTD. Monomeric superfolder GFP (sfGFP) fused with either the first 36 (N36-sfGFP) or first 48 (N48-sfGFP) residues of CsoSCA-NTD were co-expressed with synthetic carboxysomes in $E$. coli and purified to assess GFP encapsulation. Both N36-sfGFP and N48-sfGFP produced green fluorescent carboxysomes containing the fusion protein (Fig 1E), while a negative control did not. N48-sfGFP encapsulated roughly 5-fold more protein than N36-sfGFP, but also had a slightly higher expression level, indicating that multiple elements in the peptide may be needed for efficient encapsulation and/or that amount of encapsulation is by some extent controlled by availability of protein. In summary, these results demonstrate that the $\mathrm{N}$-terminus is necessary and sufficient for encapsulating CsoSCA into the carboxysome.

\section{CsoSCA interacts with Rubisco}

Previous immunogold labeling EM and biochemical assays have suggested that CsoSCA may associate with the shell but no specific interactions have been described $(18,19)$. Thus, to identify CsoSCA's interaction partner, we measured binding of un-tagged CsoSCA against all carboxysome proteins (the shell proteins CsoS1A, CsoS1B, CsoS1D, CsoS4B; the scaffolding protein CsoS2B; and Rubisco) using BLI. This screen showed that CsoSCA interacted with Rubisco, while none of the other carboxysome proteins had detectable binding above background (Fig 2A). An N-terminal truncated protein variant ( $\triangle 36 \mathrm{CsoSCA}$ ) did not bind Rubisco, confirming NTD's involvement in the interaction (SI Fig. 2A). Native-PAGE confirmed binding between CsoSCA and Rubisco and lack of binding to the major shell proteins CsoS1A and CsoS1B (SI Fig. 1D and E). Finally, co-elution of Rubisco and N48-sfGFP using size exclusion chromatography confirmed the interaction in a solution based assay (SI Fig 1C).

Concentration dependence of CsoSCA-Rubisco binding was confirmed by BLI and $K_{\mathrm{D}}$ of the interaction determined to $1.2 \mathrm{nM}\left(\left(k_{\mathrm{on}}=2.5 \times 10^{5} \mathrm{M}^{-1} \mathrm{~s}^{-1}, k_{\text {off }}=2.9 \times 10^{-4} \mathrm{~s}^{-1}\right)\right.$ (Fig 2B and Table 1). Measuring Rubisco binding to N48-sfGFP further confirmed the N48-peptide interaction $\left(K_{\mathrm{D} 1}=\right.$ $30 \mathrm{nM}, K_{\mathrm{D} 2}=80 \mathrm{nM}$; fit to a 1:2 model) (Fig 2C, Table 1 and SI Fig 1B). The 25-fold higher $K_{\mathrm{D}}$ with CsoSCA compared to N48-sfGFP is mainly an effect of a slower off-rate, demonstrating the importance of the bivalency (resulting from CsoSCA being dimeric while N48-sfGFP is monomeric) in obtaining a high affinity interaction with Rubisco. 

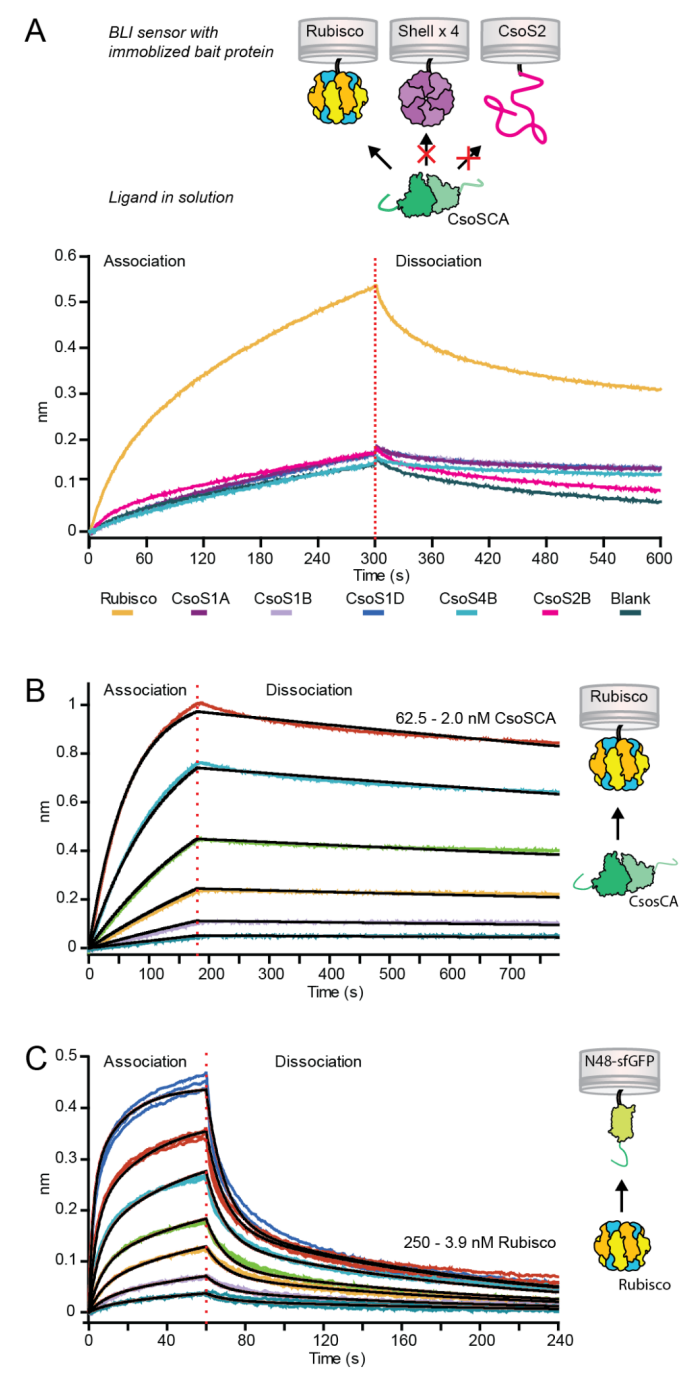

Figure 2: CsoSCA interacts with Rubisco via its $\mathbf{N}$-terminal peptide

(A) Biolayer interferometry binding screen with CsoSCA against carboxysome proteins. Binding of CsoSCA (green) were assayed against Rubisco (yellow/blue), the shell proteins; CsoS1A (purple), CsoS1B (light purple), CsoS1D (blue) and CsoS2B (light blue) and against the scaffolding protein CsoS2 (magenta). BLI responses show binding against Rubisco, while none of the other carboxysome proteins showed detectable binding. (B) BLI response from binding affinity measurement of CsoSCA against immobilized Rubisco. CsoSCA concentration ranged from 62.5 - 2.0 $\mathrm{nM}$ in a 1:2 dilution series. (C) BLI response from binding affinity measurements of Rubisco against immobilized N48-sfGFP (light green). Rubisco concentration ranged from 250 - $3.9 \mathrm{nM}$ in a 1:2 dilution series.

\section{Structure of Rubisco in complex with CsoSCA NTD peptide}

An emerging theme of CCM self-assembly is that Rubisco interacts with various CCM proteins via Short Linear Motifs (SLiMs) found in intrinsically disordered proteins/regions (IDP/Rs) (27-29). Since CsoSCA's N48-peptide appears to bind Rubisco (Fig. 2C and SI Fig. 3), we next sought to determine the structure of Rubisco in complex with this peptide using cryo-EM. In order to promote high occupancy of available binding sites, a 1000-fold excess of peptide over Rubisco was used in preparation of the sample for imaging. Through this approach, we obtained 
two subtly distinct reconstructions of Rubisco bound to the N48-peptide at $1.98 \AA$ (State-1) and $2.07 \AA$ (State-2) nominal resolution (SI Fig. 4). Of note, both reconstructions show densities for ordered waters and alternate side-chain conformers and are of the highest resolution obtained by $200 \mathrm{keV}$ cryo-EM to date (SI Fig. 4) (30). The two confirmations are highly similar (RMSD: $0.22 \AA$ ) with the most notable differences occurring in the $\beta$-sheet of the $N$-terminal domain of CbbL, in particular the confirmations of loops P37-D42 and G115-G125 (SI Fig. 5). Since both reconstructions display similar density for the N48-peptide, we do not attribute the difference in confirmation to the presence of the peptide but rather subtle "breathing" of Rubisco. For clarity, we choose to predominantly focus our discussion on the higher resolution $1.98 \AA$ structure (State-1).

In the cryo-EM reconstruction of N48-Rubisco, density corresponding to N48-peptide is located in a groove formed at the interfaces of two CbbL subunits (from two different $\mathrm{CbbL}_{2}$ dimers) and one $\mathrm{CbbS}$ (Fig. 3). The biological assembly of Rubisco is $\mathrm{CbbL}_{8} \mathrm{~S}_{8}$, resulting in eight of these binding sites per Rubisco oligomer. The peptide density is of marginally lower quality (local resolution estimate: $2.1-2.2 \AA$ ) than that of the surrounding Rubisco density. Nevertheless, we could confidently assign the density to nine residues of the N48-peptide starting at P22 (PRLDLIEQA) (Fig 3B,C). The N48-Rubisco structure is highly similar to a previous crystal structure of Rubisco (PDB: 1SVD, RMSD: $0.45 \AA$, SI Fig. 6), indicating that binding of the N48-peptide does not induce large-scale conformational changes in Rubisco.

The resolved region of the peptide starts at the bottom of the CbbS subunit (around loop D94-S99), runs downward within the groove between the two CbbL subunits and ends between $\beta$-strand S345-I347 in $\mathrm{CbbL}_{B}$ and loop P19-I29 in $\mathrm{CbbL}_{A}$, that results in a buried interface of approximately $700 \AA^{2}$. This short stretch of sequence is predicted by JPred to form an alpha-helix (SI Fig. 7A). Indeed, we observe this segment to form a single helical turn and thereafter an extended coil (Fig 3B,C). The sharp turn of the backbone introduced by P22 (the first observed residue of the bound peptide) ensures that the upstream peptide chain points outwards towards the solvent instead of clashing with $\mathrm{CbbL}$, a conformation likely essential for binding. 
bioRxiv preprint doi: https://doi.org/10.1101/2021.11.05.467472; this version posted November 5, 2021. The copyright holder for this preprint (which was not certified by peer review) is the author/funder, who has granted bioRxiv a license to display the preprint in perpetuity. It is made available under aCC-BY 4.0 International license.
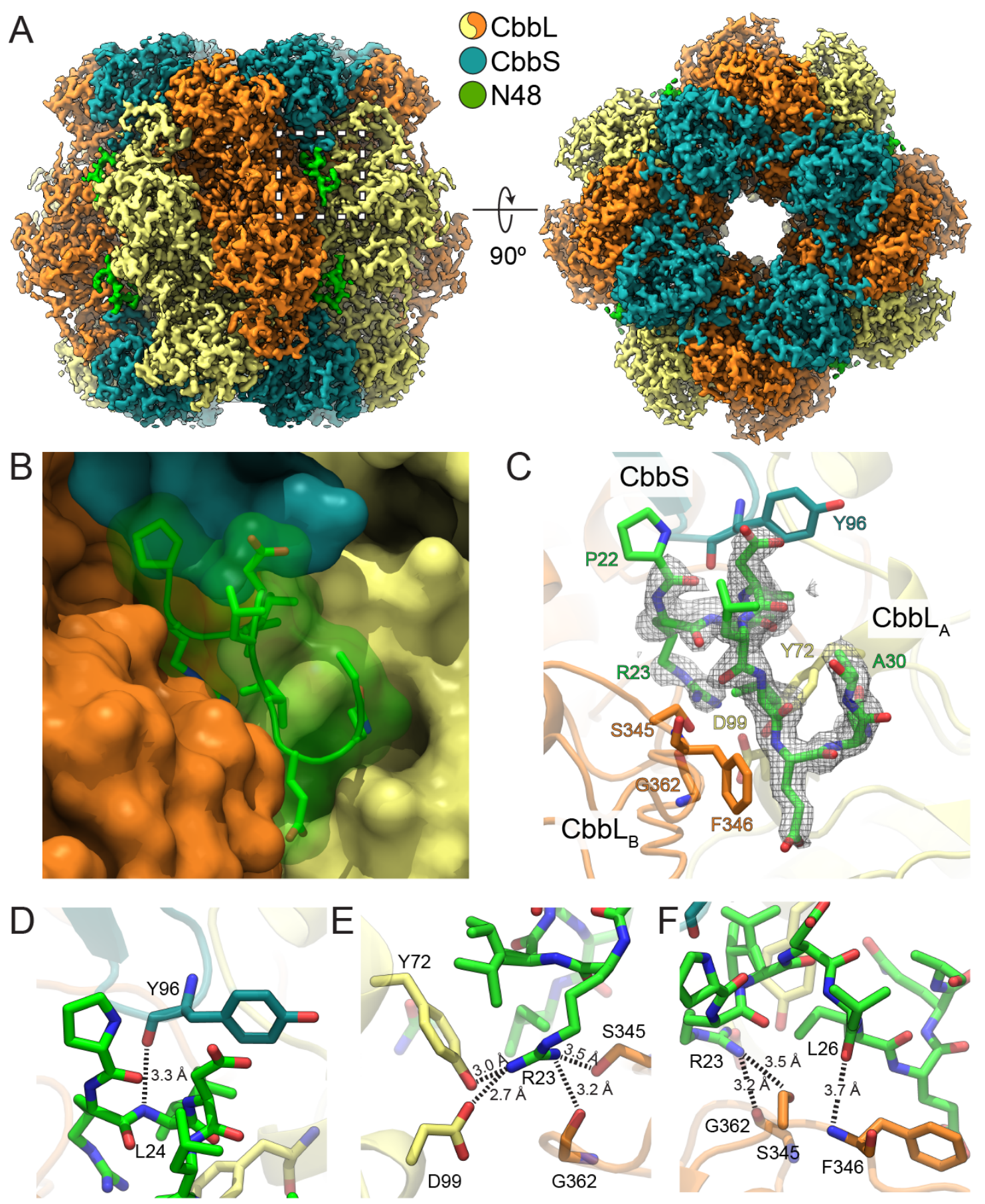

Figure 3: Structure of Rubisco with bound CsoSCA N48-peptide.

(A) Cryo-EM map of Rubisco-N48 co-complex colored by subunit with color key inset. (B) Close-up of the region boxed in $\mathrm{A}$ of the N48-peptide shown as sticks and transparent surface and Rubisco subunits shown as opaque surfaces. (C) Same view as in B with N48-peptide and interacting Rubisco residues shown as sticks. N48-peptide density is shown as a grey mesh contoured to $2 \sigma$. (D-F) Detailed polar interactions between residues of N48-peptide and Rubisco are shown as sticks with interaction depicted as dashed lines with distances in Ångströms. 


\section{Binding is predominantly mediated by a network of hydrogen bonds}

Our atomic model of the N48-Rubisco co-complex indicates that the interaction between the N48-peptide and Rubisco is largely mediated through polar interactions and, predominantly, hydrogen bonds. R23 forms an extensive network of interactions with the neighboring CbbL subunits. The side chain of R23 forms a salt-bridge with $\mathrm{D} 99\left(\mathrm{CbbL}_{\mathrm{A}}\right)$ and hydrogen bonds to the hydroxyl groups of the CbbL subunits $Y 72\left(\mathrm{CbbL}_{A}\right)$ and $\mathrm{S} 345\left(\mathrm{CbbL}_{B}\right)$, as well as to the carbonyl of G362 ( $\mathrm{CbbL}_{\mathrm{B}}$ ) (Fig 3E). The L24 amide and L26 carbonyl hydrogen bond to the carbonyl of CbbS Y96 and amide of $\mathrm{CbbL}_{B}$ F346, respectively (Fig. 3D,F).

A water-mediated hydrogen bonding network likely also contributes to peptide binding (SI Fig. 8). This putative network is predominantly built up by backbone-water interactions and consists of interactions between $\mathrm{N} 29$ and $\mathrm{CbbL}_{\mathrm{A}} \mathrm{Y} 72$ (SI Fig. 8A,B), R23 and $\mathrm{CbbL}_{\mathrm{B}}$ S345, and L26 and $\mathrm{CbbL}_{\mathrm{B}} \mathrm{F} 346$ (SI Fig. 8C). However, in the lower resolution structure (State-2) the two waters mediating the interactions between the peptide and $\mathrm{CbbL}_{\mathrm{B}}$ are not resolved (SI Fig 8D), possibly due to the slightly lower resolution of this reconstruction. Rubisco residues interacting with CsoSCA have a high conservation score among a-carboxysomal Rubiscos, but are in general not conserved in $\beta$-carboxysomal Rubiscos (SI Fig. 7B) .

To determine the relative importance of the different interactions, we measured binding kinetics with a selected set of point mutations on both the N48-peptide and Rubisco (Table 1 and SI Fig. 9). The P22A mutation resulted in a dramatic loss in binding. While a protonated CbbS D94 could potentially hydrogen bond with the amide of P22, this large effect is more likely due to P22's importance in establishing the initial alpha-helical backbone conformation of the peptide or the sharp backbone turn that is essential for binding.

Table 1: Binding constants and kinetics for CsoSCA and N48-sfGFP binding to Rubisco measured with BLI.

\begin{tabular}{|c|c|c|c|c|c|c|c|}
\hline Immobilized & In solution & $K_{\mathrm{D}}(\mathrm{nM})$ & $K_{\mathrm{D} 2}(\mathrm{nM})$ & $k_{\text {on }}\left(M^{-1} s^{-1}\right)$ & $k_{\mathrm{on} 2}\left(\mathrm{M}^{-1} \mathrm{~s}^{-1}\right)$ & $k_{\text {off }}\left(\mathrm{s}^{-1}\right)$ & $k_{\text {off2 }}\left(s^{-1}\right)$ \\
\hline \multirow[t]{2}{*}{ Rubisco } & CsoSCA-MBP & 1.2 & & $2.5 \times 10^{5}$ & & $2.9 \times 10^{-4}$ & \\
\hline & & \pm 0.1 & & $\pm 1 \times 10^{2}$ & & $\pm 2 \times 10^{-5}$ & \\
\hline \multirow[t]{2}{*}{ N48-sfGFP } & Rubisco & 33 & 80 & $2.1 \times 10^{5}$ & $1.4 \times 10^{6}$ & $6.9 \times 10^{-3}$ & $1.1 \times 10^{-1}$ \\
\hline & & \pm 0.4 & \pm 1 & $\pm 2 \times 10^{3}$ & $\pm 1 \times 10^{4}$ & $\pm 2 \times 10^{-5}$ & $\pm 5 \times 10^{-4}$ \\
\hline \multirow[t]{2}{*}{ N48-sfGFP R23A } & Rubisco & 17 & 130 & $1.9 \times 10^{5}$ & $4.6 \times 10^{5}$ & $3.3 \times 10^{-3}$ & $6.1 \times 10^{-2}$ \\
\hline & & \pm 0.2 & \pm 2 & $\pm 2 \times 10^{3}$ & $\pm 8 \times 10^{3}$ & $\pm 2 \times 10^{-5}$ & $\pm 5 \times 10^{-4}$ \\
\hline \multirow[t]{2}{*}{ N48-sfGFP } & Rubisco D99A & 400 & 1100 & $1.5 \times 10^{4}$ & $1.3 \times 10^{5}$ & $5.9 \times 10^{-3}$ & $1.5 \times 10^{-1}$ \\
\hline & & \pm 10 & \pm 14 & $\pm 4 \times 10^{2}$ & $\pm 2 \times 10^{3}$ & $\pm 4 \times 10^{-5}$ & $\pm 7 \times 10^{-4}$ \\
\hline \multirow[t]{2}{*}{ N48-sfGFP } & Rubisco Y72A & 320 & 820 & $2.8 \times 10^{4}$ & $1.8 \times 10^{5}$ & $8.9 \times 10^{-3}$ & $1.5 \times 10^{-1}$ \\
\hline & & \pm 6 & \pm 11 & $\pm 5 \times 10^{2}$ & $\pm 2 \times 10^{3}$ & $\pm 4 \times 10^{-5}$ & $\pm 8 \times 10^{-4}$ \\
\hline \multirow[t]{2}{*}{ N48-sfGFP P22A } & Rubisco & 18000 & 6500 & $2.4 \times 10^{3}$ & $6.8 \times 10^{4}$ & $4.4 \times 10^{-2}$ & $4.4 \times 10^{-1}$ \\
\hline & & \pm 3000 & \pm 400 & $\pm 4 \times 10^{2}$ & $\pm 4 \times 10^{3}$ & $\pm 3 \times 10^{-4}$ & $\pm 7 \times 10^{-3}$ \\
\hline
\end{tabular}


Despite the many interactions made by the buried peptide residue $\mathrm{R} 23$, mutation of this residue to alanine yielded roughly the same $K_{D}$-value as wild-type. However, mutation of the residues on $\mathrm{CbbL}_{\mathrm{A}}$ which interact with R23 - Y72A and D99A - resulted in a 10-fold increase in $K_{\mathrm{D}}$ (mainly an effect of slower on-rate). These results are consistent with the net contribution of interactions made by R23 to binding to be quite low, but, nevertheless, this residue adversely affects binding when these interactions are not satisfied in a buried conformation. In this context, R23 may play a role in establishing the specificity of the interaction between Rubisco and CsoSCA.

The remaining hydrogen bonds between the N48-peptide and Rubisco are mediated by backbone moieties. Furthermore, the peptide is tightly-packed in the cleft formed by Rubisco to form a buried interface comprising $700 \AA^{2}$ of the $1200 \AA^{2}$ solvent acessible surface area of the peptide. Thus, given the strong negative effect of the P22A mutant on binding, shape-complementarity between peptide and Rubisco appears critical to enable the extensive backbone-mediated hydrogen bonds and van der Waals interactions that drive binding.

\section{CsoSCA binds at the same site as CsoS2}

a-carboxysome assembly is mediated by a repetitive and disordered protein, CsoS2, which is thought to bind both Rubisco and shell proteins, thus serving as a physical scaffold bridging these two major components. We previously solved the structure of Rubisco in complex with an $\mathrm{N}$-terminal peptide derived from CsoS2 (CsoS2-N*) (27). Surprisingly, CsoSCA and CsoS2 bind at nearly the same location on Rubisco but utilize substantially different SLiMs and binding modes (Fig 4).

CsoS2-N* is largely alpha-helical and binds Rubisco by spanning over the $\mathrm{CbbL}_{2}$ dimer interface lying on top of the protein surface (Fig 4B). The complex is highly dependent on salt bridges and cation-pi interactions. In contrast, the CsoSCA peptide is bound in a conformation turned roughly $\sim 45$ degrees and with a greater fractional buried surface area for the observed peptide (approximately $700 \AA^{2}$ of $1200 \AA^{2}$ solvent acessible surface area, compared to $830 \AA^{2}$ of 2500 $\AA^{2}$ ). CsoSCA is buried deeper into the groove between the two CbbL subunits and interacts mainly via hydrogen bonds and what appears to be an ordered network of water molecules. Notably, both peptides make significant interactions with Rubisco CbbL Y72 (Fig 4B). This residue is conserved in a-carboxysome Rubisco, but not in Rubisco from $\beta$-carboxysomes or the Form II Rubisco in $H$. neapolitanus, and likely contributes to specificity. Both proteins interact with Y72 via arginines; however, in CsoS2-N* R10 is cation-pi stacked between CbbL Y72 and CbbS Y96, while in CsoSCA, R23 is positioned deeper into the structure and hydrogen bonds with CbbL Y72 and D99. Another notable feature is CbbS Y96, which in the Rubisco-CsoS2 structure is flipped $\sim 90$ degrees compared to the wild-type and Rubisco-CsoSCA structure (Fig $4 \mathrm{~B}$ ), covering the groove between the $\mathrm{CbbL}$ subunits interface and enabling the conformation necessary for cation-pi interaction.

Combined, these interactions would seemingly make it impossible for CsoSCA and CsoS2 to bind Rubisco at the same time. We have previously developed a Rubisco-CsoS2-N* fusion with all such binding sites occupied due to high local concentration of the CsoS2-N* peptide. As 
bioRxiv preprint doi: https://doi.org/10.1101/2021.11.05.467472; this version posted November 5, 2021. The copyright holder for this preprint (which was not certified by peer review) is the author/funder, who has granted bioRxiv a license to display the preprint in perpetuity. It is made available under aCC-BY 4.0 International license.

expected, BLI measurement indicated that N48-sfGFP cannot bind to Rubisco when CsoS2-N* is already present, thus confirming that CsoS2 and CsoSCA compete for the same binding site (Fig 4C).

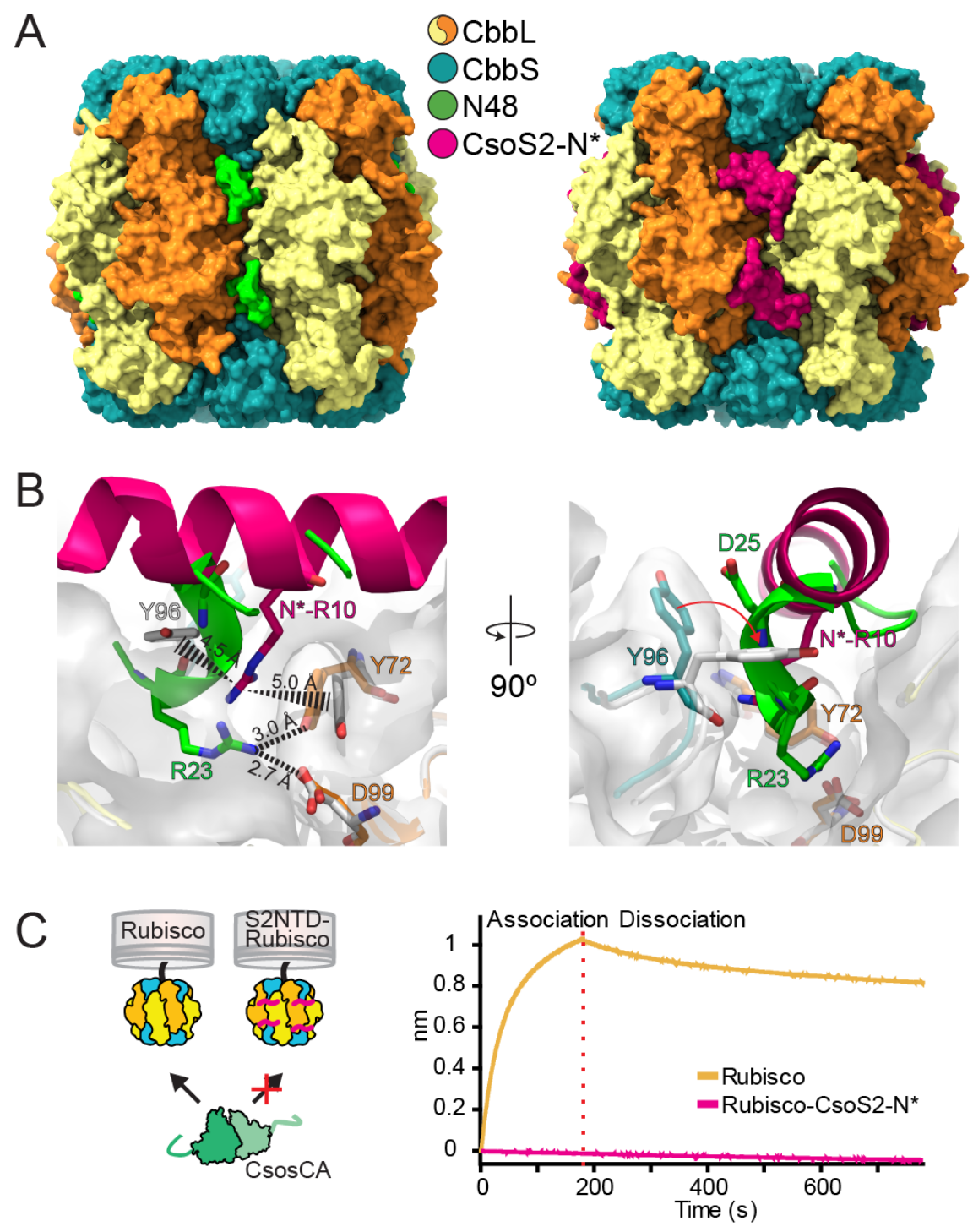

Figure 4: CsoSCA and CsoS2 bind at the same site on Rubisco.

(A) Surface representation of Rubisco with CsoSCA peptide bound and Rubisco with CsoS2 peptide bound (pdb: 6uew). (B) Zoomed view of binding site showing the different conformations of the CsoSCA and CsoS2 peptides. Peptides are shown as cartoons and detailed residues as sticks. Rubisco bound with CsoSCA N48-peptide is colored according to color key inset in (A) and Rubisco (both subunits) bound with CsoS2-N* peptide (pdb: 6uew) is colored white. White transparent surface represents the Rubisco structure which binds CsoSCA. Polar interactions are depicted as dashed lines and cation-pi stacking as dashed triangles with distances in Ångströms. (C) BLI response shows that the CsoS2 peptide fused to Rubisco passivate binding of CsosCA to Rubisco. 


\section{Discussion}

In this study, we have determined the structural basis for carbonic anhydrase encapsulation in a-carboxysomes. We found that in the model organism $H$. neapolitanus, CsoSCA and Rubisco form a previously unknown supercomplex. Through biophysical measurements and in vivo experiments we found that this complex-formation is dependent on the intrinsically disordered $\mathrm{N}$-terminal peptide of CsoSCA. The cryo-EM structure of Rubisco in complex with this peptide reveals that CsoSCA binds Rubisco at a site overlapping with that of the scaffolding protein CsoS2. Aside from its enzymatic activity, this establishes Rubisco's additional function as an interaction hub in assembly of the a-carboxysome.

\section{Updated model of $\alpha$-carboxysome architecture and assembly}

The intrinsically disordered repeat protein CsoS2 acts as a scaffolding between shell and Rubisco and orchestrates the assembly $(31,32)$ of the a-carboxysome. We recently discovered that a repeat of conserved SLiMs (four repeats/protein) found in the N-terminal portion of CsoS2 binds Rubisco and is essential for carboxysome formation (26). In contrast to earlier observations, we demonstrate here that the carboxysomal carbonic anhydrase (CsoSCA) is not directly associated with the shell, but is recruited to the carboxysome via association with Rubisco. We further show that CsoSCA's N-terminal targeting peptide and CsoS2 binds at the same site on Rubisco. Figure 5 presents our current model of $\alpha$-carboxysome assembly.

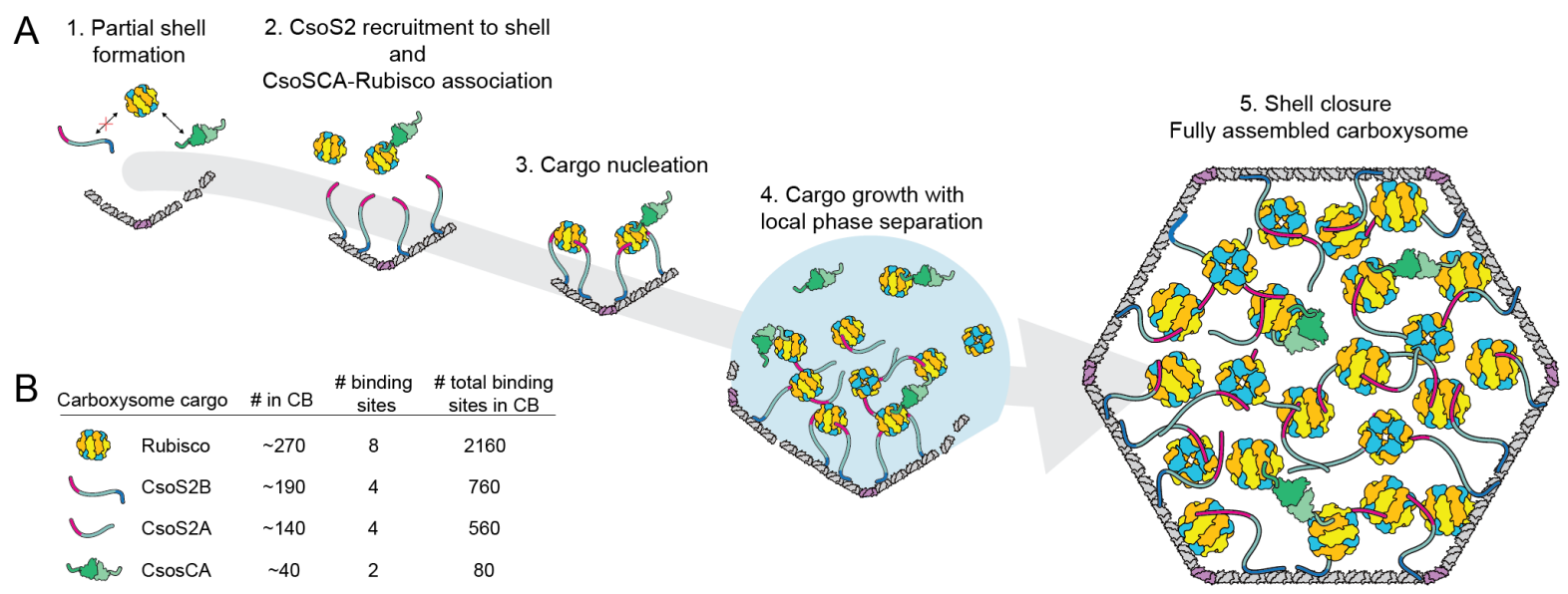

Figure 5: Updated model for carboxysome assembly.

(A) Schematic model of a-carboxysome assembly in which CsoSCA recruits to the carboxysome via interaction with Rubisco. This is an updated version of the model presented in Oltrogge et al., 2020, (26) with recruitment of CsoSCA added. Current knowledge does not allow us to distinguish between whether CsoSCA associates to Rubisco in step 2 or if association occurs in the phase separated condensate in step 4. The fully assembled carboxysome in step 5 shows a stoichiometric accurate - with respect to cargo proteins - version of the $\alpha$-carboxysome. (B) Average number of cargo proteins present in an a-carboxysome (33), number of binding sites per oligomeric form of cargo protein and total number of binding sites per carboxysome. 
Previous work has shown that, on average, there are 270, 330 and 40 copies of Rubisco, CsoS2 and CsoSCA in a typical $H$. neapolitanus carboxysome, respectively (Fig. 5B) (33). The roughly 1300 CsoS2 and 80 CsoSCA Rubisco binding motifs per carboxysome set an upper boundary of occupancy for the binding sites of Rubisco ( 2200 sites/carboxysome). Assuming all CsoS2 and CsoSCA motifs engage in binding, roughly $60 \%$ of Rubisco sites would be occupied by CsoS2 while considerably less, $\sim 3.5 \%$, would be occupied by CsoSCA. Although this assumes that all Rubisco sites are accessible and that all CsoS2 and CsoSCA motifs bind, both assumptions of which could be incorrect, such a calculation indicates there is likely a surplus of Rubisco sites available for binding. It has previously been shown that CsoSCA mRNA levels are lower compared to other carboxysome genes (34), suggesting that the amount of encapsulated CsoSCA is likely regulated by protein expression level rather than by competing for binding site occupancy with CsoS2. Due to the need for tight regulation of CA activity outside of the carboxysome, efficient encapsulation is vital (17) and a scenario where CsoSCA had to compete for binding could pose a physiological problem.

One specific unknown is the importance of CsoSCA bivalency imparted by its homodimeric structure and the resulting mode of interaction with Rubisco. The significant slower dissociation rate of full-length CsoSCA (bivalent) compared to the N48-peptide (monovalent) implies an importance of multivalent/bivalent protein-protein interactions (Table 1 and Figure 2B,C), a feature commonly observed for other IDP/R involved in phase separation (35). In terms of binding mode, bivalent binding of full-length CsoSCA could occur either between two binding sites on the same Rubisco or between two sites on different Rubisco molecules. The relatively short stretch of IDR sequence before the Rubisco binding motif and the rigidity of the folded domains presumably constrains possible binding conformations where CsoSCA binds on top or on the side in a 1:1 CsoSCA-Rubisco complex (SI Fig. 10). Alternatively, CsoSCA could cross-link two Rubisco molecules (SI Fig. 10). Current data cannot exclude these different possibilities and further structural and biochemical experiments are needed to shed light on CsoSCA's quaternary binding conformation in the native context of carboxysomes.

\section{Plasticity of the Rubisco binding motif}

We could not identify a consensus Rubisco SLiM binding motif across NTD sequences in CsoSCA homologs. The binding element identified in $H$. neapolitanus CsoSCA (PRLDLIEQA) is present in its most closely related homolog (Halothiobacillus sp. LS2) but is not conserved across species (further discussed in SI Figure 11A and B). Many Prolines are followed by R or $\mathrm{xR}$, but, overall, the proteobacteria clade contains no convincing conserved motifs. In the cyanobacterial clade, a PTAPx[R/K]R motif is present in $87 \%$ of the sequences suggesting a possible binding motif among cyanobacteria.

Surprisingly, a handful of cyanobacterial CsoSCA sequences from the Procloroccocos genus contain the Rubisco-binding motif found in CsoS2 (RxxxxxRRxxxxxxGK) (SI Figure 11A), suggesting an evolutionary relationship. The lack of a consensus motif in CsoSCA homologs, coupled with the fact that CsoSCA and CsoS2 bind at the same site but with different mechanisms, reveals an evolutionary plasticity in SLiM sequence space. Across the various 
microbes in this phylogeny, we hypothesize that $\mathrm{CA}$ is recruited to its respective a-carboxysomes by the observed, versatile Rubisco binding site and does so using diverse SLiM sequences.

\section{Rubisco as an interaction hub in biophysical CCM's}

Recent work on both bacterial carboxysomes and algal pyrenoids suggests that Rubisco itself plays a role as an interaction hub in the ultrastructural organization of CCMs. It is now clear that not only does Rubisco interact with scaffolding proteins as a means to condensate Rubisco and form these confined $\mathrm{CO}_{2}$-fixing organelles, it also recruits auxiliary proteins, such as CAs and activases, needed for the CCM to function. In the bacterial carboxysomal a-system, we have demonstrated that Rubisco binds the intrinsically disordered proteins CsoS2 (26) and CsoSCA. Additionally, it also binds the Rubisco activase CbbQO, likely via CbbO's von Willebrand factor A domain $(36,37)$ The $\beta$-carboxysomal Rubisco binds its interaction partners, the scaffolding protein CcmM (38) (39), and the Rubisco activase Rca $(40,41)$, via a folded domain resembling the small subunit of Rubisco. Despite convergent evolution, a notable similarity between both carboxysome systems is the binding site on Rubisco. In known cases (except for CbbQO), the interactor binds at the same patch on Rubisco and makes contact with two different $\mathrm{CbbL}_{2}$ dimers and one CbbS. This likely ensures that the binding partner only interacts with fully assembled $\mathrm{CbbL}_{8} \mathrm{~S}_{8}$ Rubisco's during the assembly process.

In contrast to these bacterial systems, in the model algae Chlamydomonas reinhardtii a repeat SLiM in the disordered scaffolding protein EPYC is essential for pyrenoid formation $(42,43)$ and binds on top of the small subunit via salt bridges and a hydrophobic interface (28). The sequence motif is shared among many pyrenoid proteins, suggesting a mechanism for protein targeting as well as more broadly organizing pyrenoid ultrastructure (29). The versatility in binding motif and binding site, and the convergent function of diverse Rubisco as a hub of interaction implies this might be a general feature, which raises a final question. Do Form IB plant Rubiscos engage in similar protein-protein interactions and do other Rubisco Forms also function as interaction hubs?

In summary, this work advances our understanding of carboxysome biogenesis, puts a focus on the essential carbonic anhydrase and the central role of Rubisco as a hub protein. This provides critical findings for engineering the carboxysome based CCM into e.g. crops and industrially relevant microorganisms for improved growth and yields. More broadly, we hope that the findings presented here will advance our understanding of bacterial microcompartments and promote development of their many potential biotechnological applications. 


\section{Materials and Methods}

\section{Bioinformatics}

Protein sequences assigned CsoSCA (pfam08936) from all finished and permanent draft bacterial genomes available in the Integrated Microbial Genomes and Microbiomes database (IMG) (44) were collected on December 12, 2019 and curated to only include proteins in an a-carboxysome operon (containing CbbL/S, CsoS2 and shell hexamers and pentamers) (412 genes). Thereafter redundancy was reduced by removing sequences with $>98 \%$ identity using Jalview and sequences were manually curated to remove incomplete sequences, resulting in 222 sequences in the final CsoSCA dataset (SI Table 1). Sequences were aligned using MUSCLE (45). Resulting multiple sequence alignment (MSA) was used to calculate conservation score (46). Tree was built using IQ-TREE web server (47) and visualized using iTOL (48). Protein disorder were predicted for a subset of the dataset, including $H$. neapolitanus CsoSCA, using the DISOPRED3 algorithm (49). Conservation of CsoSCA NTD Rubisco binding motif was analysed using The MEME Suite (50) (SI Table 1). MSA of a-carboxysomal Form IA Rubiscos (135 cbbL and $132 \mathrm{cbbS}$ sequences) and $\beta$-carboxysomal Form IB Rubiscos (211 cbbL and 207 cbbS sequences) was constructed (SI Table 1) using, MUSCLE and visualised with WebLogo. Secondary structure prediction of NTD sequence were performed using Jpred4 (51)

\section{Protein expression and purification}

Expression plasmids were transformed into either E. coli BL21-A1 or BW25113 cells (SI Table 2). For protein expression, cells harboring appropriate plasmids (Table 2) were grown at $37^{\circ} \mathrm{C}$ in LB-medium supplemented with appropriate antibiotics. All Rubisco constructs were co-transformed with pGro7 for co-expression of GroEL/ES. At $\mathrm{OD}_{600}=0.4-0.6$ the temperature was decreased to $18{ }^{\circ} \mathrm{C}$ and expression induced by addition of $0.08 \% \mathrm{~L}$-arabinose, $0.1 \mu \mathrm{M}$ anhydrotetracycline (ATc) or $0.5 \mathrm{mM}$ IPTG, see SI Table 2. Cells were grown overnight, harvested by centrifugation at $5,000 \times g$ and frozen at $-20^{\circ} \mathrm{C}$ until use. All proteins were purified with either His-tag och Strep-tag affinity purifications. Bacterial pellets were thawed and resuspended in appropriate binding buffer supplemented with $0.2 \mathrm{mM}$ phenylmethanesulfonyl fluoride (PMSF), $0.1 \mathrm{ml} / \mathrm{ml}$ lysozyme and $0.1 \mathrm{ul} / \mathrm{ml}$ benzonase and lysed by three passes through a homogeniser (Avestin EmulsiFlex-C3) or by chemical lysis by diluting the resuspension 1:1 in B-PER II (Thermo Fisher) an incubating in RT for 30 min on a rocking table. Lysed cells were clarified by centrifugation at $27,000 \times g$ for $45 \mathrm{~min}$.

His-tag purification: Clarified lysate was applied to a $5 \mathrm{ml}$ HisTrap FF column (GE Healthcare) equilibrated with His-binding buffer (50 mM Tris, $300 \mathrm{mM} \mathrm{NaCl}, 20 \mathrm{mM}$ imidazole, pH 8.0) using a syringe pump at a flow rate of $5 \mathrm{ml} / \mathrm{ml}$. Unspecific bound proteins were washed away with His-buffer containing $60 \mathrm{mM}$ imidazole until A280 reached a stable baseline, and protein thereafter eluted with His-buffer containing $300 \mathrm{mM}$ imidazole. Strep-tag purification: Clarified lysate was applied to a $5 \mathrm{ml}$ StrepTrap column (GE Healthcare) equilibrated with Strep-buffer (50 mM Tris, $300 \mathrm{mM} \mathrm{NaCl}, \mathrm{pH}$ 8.0). Unspecific bound proteins were washed away with 
Strep-buffer until A280 reached a stable background, and protein thereafter eluted with Strep-buffer supplemented with $2.5 \mathrm{mM}$ d-desthiobiotin (Sigma-Aldrich). All purified proteins were concentrated to appropriate concentration using $30 \mathrm{kDa} \mathrm{Mw}$ cut-off centrifugal filters (Amicon) and buffer exchanged into $50 \mathrm{mM}$ Tris, $150 \mathrm{mM} \mathrm{NaCl}, \mathrm{pH} 7.5$ using 10DG Desalting Columns (Bio-Rad). Untagged CsoSCA: Untagged CsoSCA were purified as a His-SUMO fusion as outlined above. After elution from HisTrap column His-SUMO-CsoSCA was concentrated from $10 \mathrm{ml}$ to $3 \mathrm{ml}$ and buffer exchange into $50 \mathrm{mM}$ Tris, $300 \mathrm{mM} \mathrm{NaCl}, 10 \%$ glycerol $\mathrm{pH}$ 8.0. The SUMO-tag was thereafter cleaved by addition of 1:200 molar ratio of Ulp-protease:His-SUMO-CsoSCA and 1 hour incubation. Cleaved protein mix was applied to a $1 \mathrm{ml}$ HiTrap column to remove His-SUMO and residual uncleaved His-SUMO-CsoSCA. This resulted in a pure and untagged CsoSCA. Since full-length CsoSCA is prone to crash out in high protein concentrations care was taken to not exceed CsoSCA protein concentration above $1 \mathrm{mg} / \mathrm{ml}$. CsoSCA-MBP: After His-tag purification protein was subsequently purified by size exclusion chromatography using a Superose 6 Increase 10/300 column (GE Healthcare). Protein purities were assessed by SDS-page and were in general $>95 \%$ pure. Protein concentrations were determined by $A_{280}$ and the theoretically calculated extinction coefficient (ProtParam). All purifications were carried out at $4{ }^{\circ} \mathrm{C}$. For storage, proteins were made to $10 \%$ $(\mathrm{w} / \mathrm{v})$ glycerol, flash-frozen in liquid nitrogen and stored in $-800^{\circ} \mathrm{C}$.

\section{Growth phenotypes of $\mathrm{H}$. neapolitanus csoSCA mutants}

Generation of $H$. neapolitanus $\triangle$ csoSCA and of WTcsoSCA and $\triangle 48$ csoSCA mutant complementations: csoSCA was knocked out by transforming a plasmid containing a spectinomycin cassette with $1 \mathrm{~KB}$ homology arms adjacent to csoSCA gene. Colonies that grew on spectinomycin were verified by colony PCR and Sanger Sequencing to verify a clean knockout. CsoSCA mutant complementations ( $\triangle \operatorname{csoSCA}+W T \operatorname{csoSCA} \triangle \Delta \operatorname{csoSCA}+\triangle 48 \operatorname{csoSCA})$ were generated by transformation and concomitant genomic integration via homologous recombination into $H$. neapolitanus NS2 neutral site and verified by colony PCR and Sanger sequencing.

$H$. nea growth assays: Pre-cultures of WT $H$. neapolitanus and $H$. neapolitanus $\triangle$ csoSCA were grown in DSMZ68 at $5 \% \mathrm{CO}_{2} . \triangle \operatorname{csoSCA}$ transformed with wild-type csoSCA or the N-terminal truncation $\triangle 48 C$ soSCA were cultured in the same conditions with the addition of $1 \mu \mathrm{M}$ IPTG to induce CA expression. All precultures were grown with the appropriate antibiotics. Upon reaching log phase, cultures were spun down for 15 minutes at 4,000 $\times$ g. Pellets were then resuspended in $1 \mathrm{~mL}$ DSMZ68 without thiosulfate and $\mathrm{pH}$ indicator. Cultures were transferred into $1.7 \mathrm{~mL}$ eppendorf tubes and centrifuged at $4,000 \times \mathrm{g}$ for 8 minutes. This wash step was repeated twice before the cultures were diluted $5 x$ and then normalized to a cell density of 0.1 OD600. Normalized cultures were serially diluted in $10 \mathrm{x}$ steps from $10^{-1}$ to $10^{-8}$ OD600. Resulting titers were spotted in $3 \mu$ volumes onto plates with appropriate antibiotics in $5 \% \mathrm{CO}_{2}$ and ambient air; strains expressing complemented WTcsoSCA or $\triangle 48 c s o S C A$ were plated on plates containing $1 \mu \mathrm{M}$ IPTG. Strains were allowed to grow for 4 days. All strains were plated in biological and technical triplicate. 


\section{Carboxysome purifications}

Small scale enrichment of carboxysomes: $100 \mathrm{ml}$ of E. coli BW25113 cells harbouring pHnCB10 (plasmid for homologous expression of $H$. neapolitanus a-carboxysomes in $E$. coli), containing either wildtype, $\Delta 36$ or $\Delta 48$ truncation of flag-tagged CsoSCA, were grown at $30{ }^{\circ} \mathrm{C}$ in LB-medium supplemented with appropriate antibiotics (SI Table 1). At $\mathrm{OD}_{600}=0.4-0.6$ the expression was induced by addition of $0.5 \mathrm{mM}$ IPTG, cells grown for $4 \mathrm{~h}$, harvested by centrifugation at $5,000 \times g$ and frozen at $-20{ }^{\circ} \mathrm{C}$ until use. To enrich carboxysomes, cells from $100 \mathrm{ml}$ of culture were resuspended and chemically lysed for $30 \mathrm{~min}$ in $6 \mathrm{ml}$ of B-PER II (Thermo Fisher) diluted to $1 \mathrm{x}$ with TEMB buffer $(10 \mathrm{mM}$ Tris $\mathrm{pH} 8.0,10 \mathrm{mM} \mathrm{MgCl} 2,20 \mathrm{mM}$ $\mathrm{NaHCO}_{3}$ and $1 \mathrm{mM}$ EDTA) supplemented with $0.1 \mathrm{mg} / \mathrm{mL}$ lysozyme, $1 \mathrm{mM} \mathrm{PMSF}$ and $0.1 \mathrm{ul}$ of benzonase/mL (Sigma-Aldrich). Lysed cells were centrifuged 12,000 x $g$ for 15 min to remove cell debris. The clarified lysate was centrifuged $40,000 \times \mathrm{g}$ for $30 \mathrm{~min}$ and the enriched carboxysome fraction were thereafter resuspended in $200 \mathrm{ul}$ of TEMB.

Full scale purification of carboxysomes co-expressed with CsoSCA-sfGFP fusions: $1 \mathrm{~L}$ of $E$. coli BW25113 cells co-transformed with pHnCB9 (pHnCB10 lacking the gene for csoSCA) and pFA-plasmid containing a CsoSCA variants fused to sfGFP (N36-sfGFP, N48-sfGFP or CsoSCA-sfGFP) were grown at $37{ }^{\circ} \mathrm{C}$ in LB-medium supplemented with appropriate antibiotics (SI Table 2). At $\mathrm{OD}_{600}=0.4-0.6$ the expression was induced by addition of $0.5 \mathrm{mM}$ IPTG and $0.1 \mu \mathrm{M}$ aTc, temperature decreased to $18^{\circ} \mathrm{C}$ and grown o/n. Thereafter cells were harvested by centrifugation at $5,000 \times g$ and frozen at $-20{ }^{\circ} \mathrm{C}$ until use. To purify carboxysomes, cells were chemically lysed for $30 \mathrm{~min}$ under mild shaking in B-PER II (Thermo Fisher) diluted to $1 \mathrm{x}$ with TEMB buffer supplemented with $0.1 \mathrm{mg} / \mathrm{mL}$ lysozyme, $1 \mathrm{mM} \mathrm{PMSF}$ and $0.1 \mathrm{ul}$ of benzonase $/ \mathrm{mL}$ (Sigma-Aldrich). Lysed cells were centrifuged $12,000 \times \mathrm{g}$ for $15 \mathrm{~min}$ to remove cell debris. The clarified lysate was centrifuged $40,000 \times \mathrm{g}$ for $30 \mathrm{~min}$ to pellet carboxysomes and obtained pellets were gently resuspended in $1.5 \mathrm{~mL}$ TEMB buffer. Resuspended pellets were loaded on top of a $25 \mathrm{~mL} 10-50 \%$ sucrose step gradient $(10,20,30,40$ and $50 \% \mathrm{w} / \mathrm{v}$ sucrose, made in TEMB buffer) and ultracentrifuged at $105,000 \times \mathrm{g}$ for $35 \mathrm{~min}$ (SW 32 Ti Swinging-bucket, Beckman Coulter). Gradients were fractionated, analysed by SDS-PAGE and carboxysome containing fractions pooled and ultracentrifuged $100,000 \times g$ for $90 \mathrm{~min}$. Resulting pellets were gently resuspended in TEMB to obtain the final purified carboxysome sample.

Final carboxysome samples and as well as the lysate were analysed for presence of CsoSCA or sfGFP-fusion protein by SDS-page (4-20\% Mini-PROTEAN® TGX ${ }^{\mathrm{TM}}$ Precast Protein Gels (Bio-Rad)), western-blot and GFP-fluorescence. For western blotting, proteins from SDS-page gels were transferred to nitrocellulose membranes using the Trans-Blot Turbo system (Bio-Rad). Membranes were blocked with $5 \%(\mathrm{w} / \mathrm{v})$ non-fat dry milk in phosphate-buffered saline (PBS), $0.1 \%(\mathrm{v} / \mathrm{v})$ Triton X-100 for one hour at room temperature. Immunolabeling of Flag-tag was done overnight in $4{ }^{\circ} \mathrm{C}$ in the above mentioned buffer containing a 1:5,000 dilution of a monoclonal anti-Flag horseradish peroxidase conjugated antibody (Sigma). Membranes were washed 3x10 minutes with PBS, $0.1 \%(\mathrm{v} / \mathrm{v})$ Triton $\mathrm{X}-100$, and blots were thereafter developed using SuperSignal West Pico Chemiluminescent Substrate (ThermoFisher) according to manufacturer's procedure. Gels and western blots were imaged with ChemiDoc ${ }^{\mathrm{TM}} \mathrm{XRS}+\mathrm{System}$ 
(Bio-Rad). Fluorescence of sfGFP-samples was quantified using a Infinite M-1000 plate reader (Tecan), and normalized against fluorescence of CsoSCA-sfGFP sample.

\section{Biolayer interferometry}

Protein-protein interactions were measured by Biolayer interferometry (BLI) using an Octet RED384 (Forte Bio). Experimental binding sequence used was: Loading bait 60-240 s, buffer wash $60 \mathrm{~s}$, prey association and prey dissociation (followed by sensor regeneration for Ni-NTA sensors). CsoSCA binding screen: Bait proteins were immobilized on Octet $囚$ Ni-NTA Biosensors (Forte Bio) via terminal His-tag using $1.5-8 \mu \mathrm{g} / \mathrm{ml}$ of protein. To avoid tiling of shell proteins on the sensor surface SUMO-fusions were used for CsoS1A, 1B, 1D and 4B (52). $1 \mu \mathrm{M}$ untagged CsoSCA was used as soluble prey protein. Binding assay was performed in a final buffer of $145 \mathrm{mM}$ Tris $\mathrm{pH} 7.5,30 \mathrm{mM} \mathrm{NaCl}, 1.0 \mathrm{mM}$ TCEP and $0.01 \%$ Triton X100. After a run Ni-NTA sensors were regenerated in $50 \mathrm{mM}$ Tris $\mathrm{pH} 8.0,300 \mathrm{mM} \mathrm{NaCl}, 300 \mathrm{mM}$ imidazol, $0.05 \%(\mathrm{w} / \mathrm{v})$ SDS and experiment performed in triplicate. N48-sfGFP vs. Rubisco: Assays were performed as described above using $5 \mu \mathrm{g} / \mathrm{ml} \mathrm{N48-sfGFP-His} \mathrm{as} \mathrm{bait} \mathrm{and} \mathrm{varied} \mathrm{concentration} \mathrm{of}$ strep-Rubisco as prey (wt: 250 - $3.9 \mathrm{nM}$, for mutants se Figure SI X) in $25 \mathrm{mM}$ Tris pH 7.5, 70 $\mathrm{mM} \mathrm{NaCl}$ and $0.01 \%$ Triton $\mathrm{X} 100$ and performed in triplicate of duplicates. When indicated, point mutants of N48-sfGFP or Rubisco were used. Rubisco vs. CsoSCA: $2.5 \mathrm{mg} / \mathrm{ml}$ of biotinylated Rubisco was immobilized as bait protein on Octet ${ }^{\circledR}$ Streptavidin Biosensors (Forte Bio) and 62.5 - $2.0 \mathrm{nM}$ of C-terminal MBP tagged CsoSCA was used as prey. Experiment was performed in triplicate in $25 \mathrm{mM}$ Tris $\mathrm{pH} 7.5,125 \mathrm{mM} \mathrm{NaCl}$ and $0.01 \%$ Triton $\mathrm{X} 100$. Rubisco vs. N48 peptide: Biotinylated Rubisco was used as bait and N48 peptide (100, 50 and $10 \mathrm{ul}$ ) as prey in a final buffer of $25 \mathrm{mM}$ Tris, $\mathrm{pH} 7.5,85 \mathrm{mM} \mathrm{NaCl}$ and $0.01 \%$ Triton X100. Binding and kinetic constants were extracted using the Data Analysis HT 10.0.00.44 software in the Octet Forte Bio package. N48-sfGFP vs. Rubisco were fitted to a 1:2 (Bivalent Analyte) binding model and Rubisco vs. CsoSCA to a $1: 1$ binding model.

\section{Size exclusion chromatography analysis of N48-sfGFP:Rubisco co-complex}

Purified Rubisco-strep and N48-sfGFP samples from above were exchanged into moderate-salt buffer $(20 \mathrm{mM}$ Tris, pH 7.5, $150 \mathrm{mM} \mathrm{NaCl}$ ) using Zeba desalting columns. For co-complexing, the protein samples were mixed at an 32:1 ratio (N48-sfGFP:CbbL $\mathrm{S}_{8}$ ) and incubated briefly on ice prior to injection over a 3.2/300 Superose 6 Increase column equilibrated in $20 \mathrm{mM}$ Tris, pH $7.5,150 \mathrm{mM} \mathrm{NaCl}$ at $4^{\circ} \mathrm{C}$. The column was eluted isocratically in the same buffer with elution of total protein monitored by absorbance at $280 \mathrm{~nm}$ and elution of sfGFP-containing fractions monitored by absorbance at $485 \mathrm{~nm}$.

\section{Cryo-EM of CsoSCA-Rubisco complex}

$0.5 \mu \mathrm{M}$ Rubisco-strep was mixed with $0.5 \mathrm{mM}$ of N48-peptide (CsoSCA residue 1-50) in $25 \mathrm{mM}$ Tris $\mathrm{pH} 7.5,80 \mathrm{mM} \mathrm{NaCl}$ containing $2 \%$ glycerol, incubated for $20 \mathrm{~min}$ at room temperature and thereafter stored on ice. $3.5 \mu \mathrm{L}$ of this sample was deposited onto freshly glow-discharged (PELCO easiGlow), Quantifoil R 1.2/1.3 200 mesh Copper TEM grids (Quantifoil Microtools) and blotted for $3 \mathrm{sec}$ using a Mark IV Vitrobot (FEI) after a 30 second delay under $100 \%$ humidity at $4{ }^{\circ} \mathrm{C}$ conditions before freezing in liquid ethane. The complex was visualized in a Talos Arctica 
(Thermo Fisher Scientific) operating at $200 \mathrm{keV}$, and equipped with a K3 Summit director electron detector (Gatan) in super-resolution CDS mode at 57,000x, corresponding to a pixel size of $0.69 \AA$. In total, 5,742 movies were acquired with the aid of SerialEM * using a defocus range between -0.6 to $-1.8 \mu \mathrm{m}$ and a $3 \times 3$ multishot image shift pattern. All movies consisted of 50 frames with a total dose of $50 \mathrm{e}-/ \AA^{2}$. The data collection was monitored using on-the-fly processing in cryoSPARC live (Structura Biotechnology Inc., https://cryosparc.com/live) (53) to monitor microscope performance, micrograph quality, and orientation distribution of the particles on the grid

\section{Image Processing}

Super-resolution electron micrograph movies were aligned using MOTIONCOR2 (54) from within RELION 3.1 or using the CPU implementation of motion correction within RELION 3.1. CTF estimation was performed using CTFFIND 4.1 (55) from within RELION 3.1. Micrographs were inspected to remove poor-quality images, resulting in the higher quality selection of 3,932 micrographs. All further processing was done from within RELION 3.1.

Laplacian-of-Gaussian auto-picking was used on a subset of 200 micrographs to pick approximately 75,000 particles. These particles were extracted from the micrographs with a pixel size of 2.77 Angstrom and a box size of 90 pixels. 2D classification was then used to generate a higher quality subset of particles that were used to generate an initial 3D model by way of Stochastic Gradient Descent. 3D classification of this higher quality subset of particles gave us a good quality 3D reference that was then used as a 3D template.

Approximately $1,300,000$ particles were picked from the 3932 micrographs using our 3D reference before being extracted with a pixel size of 2.77 and a box size of 90 pixels. The particles were subjected to 3D classification applying D4 symmetry and a soft circular mask and the best-looking classes comprised of 358,785 particles were selected. The particles were then re-extracted with a pixel size of 1.37 Ångstrom and a box size of 180 pixels before undergoing another round of 3D classification. Again, the best classes comprised of 290,762 particles were selected. The particles were re-extracted with a pixel size of 0.91 Ångstrom and a box size of 312 pixels before being subjected to 3D auto-refinement. The refined particles were then 3D classified without additional image alignment and the best classes comprised of 262,882 particles were selected. These particles underwent CTF refinement and Bayesian polishing before being extracted with a larger 410 pixel size box. A few more rounds of 3D classification and $3 \mathrm{D}$ refinement, while selecting only the best classes left us with a homogeneous set of 79,562 particles. 3D refinement of this particle set gives a final resolution of 1.98 Ångstom at Fourier shell correlation $(F S C)=0.143$. RELION 3.1 reports a b-factor of about $-36 \AA^{2}$ when sharpened with a soft mask.

\section{Coordinate model building and refinement}

The coordinate models for the two $H$. neapolitanus Rubisco-CsoSCA N48-peptide maps were built and refined similarly using a combination of COOT-v0.9.1 (56) and PHENIX-v1.19.1-4122 (57). Maps for this process were obtained by combination of the respective half-maps without filtering. Maps were molecular weight-based density modified and sharpened with phenix.resolve_cryo_em and phenix.auto_sharpen $(58,59)$, respectively. For ease of handling, the maps were reboxed to $160 v^{3}$ (about $145 \AA^{3}$ ) for further use. Chains $A(C b b L)$ and $D$ 
(CbbS) from the Hnea Rubisco-CsoS2 N*-peptide co-crystal structure (PDB ID: 6UEW) (27), stripped of all ligands, were used as initial models for both maps. The initial models were rigid-body docked and manually reworked to fit the maps in COOT and the resolved portion of CsoSCA N48-peptide built de novo. An initial round of phenix.real_space_refinement (60) was performed on models consisting of all asymmetric units with NCS constraints enforced, as well as default target bond length and angle restraints, but without secondary structure, rotamer, nor Ramachandran restraints. Putative ordered water molecules were then placed interactively in COOT using maps thresholded at $2 \boldsymbol{\sigma}$ based on presence of at least 2 hydrogen bonding partners and the occurrence of the density in both half-maps. Additional rounds of phenix.real_space_refinement and manual adjustment in COOT were performed as described above to yield the final coordinate models. For the higher resolution map (State-1), residues V3-E457 of CbbL were modelled with residues V324-E329 truncated to the $\mathrm{C}_{\beta}$ atoms due to poor side-chain density in this region. Similarly, for the lower resolution map (State-2), residues V3-E457 of CbbL were modelled with residues $\mathrm{H} 291-\mathrm{H} 300$ and V323-D331 truncated to the $\mathrm{C}_{\beta}$ atoms. The CbbS and N48-peptide density for both maps were modelled with residues M4-N110 and P22-A30, respectively.

\section{Visualization and structural analysis}

Structural figures were prepared using a combination of PyMOL-v2.5 (Schrödinger, LLC.) and ChimeraX-v1.2.1 (61). Interface analysis to identify interacting residues and to calculate buried surface area was performed using the ePISA-v1.52 web server (62).

\section{Data availability}

Cryo-EM maps (sharpened, full, and unfiltered halves) and masks have been deposited with the Electron Microscopy Data Bank, and the corresponding atomic coordinate models deposited with the Protein Data Bank for Rubisco-N50 peptide State-1 (EMD-25201, PDB-7SMK) and State-2 (EMD-25228, PDB-7SNV).

\section{Acknowledgment}

We thank Paul Tobias for computational systems support and Luis Valentin for bioinformatics advice. We are grateful to Sacha Pulsford, Benedict Long, Avi Flamholz, Luke Oltrogge, Julia Borden and Nikoleta Vogiatzi for advice and helpful discussion regarding the project and manuscript. Structural data were collected at Cal-Cryo at the California Institute for Quantitative Biosciences (QB3) of the University of California Berkeley. Molecular graphics and analyses was performed in part with UCSF ChimeraX, developed by the Resource for Biocomputing, Visualization, and Informatics at the University of California, San Francisco, with support from National Institutes of Health R01-GM129325 and the Office of Cyber Infrastructure and Computational Biology, National Institute of Allergy and Infectious Diseases. C.B. was partially supported by an International Postdoctoral grant from the Swedish Research Council (637-2014-6914). T.L. was supported by a NSF Graduate Research Fellowship (DGE 1106400). 
This work was funded by a grant from the U.S. Department of Energy (DE-SC00016240) to D.F.S.

\section{Author contributions}

C.B. and D.F.S. designed the research. C.B., E.D. T.G.L. M.D.L and S.R.S. conducted the experiments. J.P.R. and T.G.L. solved the structure. C.B., T.G.L and D.F.S. wrote the manuscript with input and comments from all authors.

\section{References}

1. M. R. Badger, G. D. Price, The role of carbonic anhydrase in photosynthesis. Annu. Rev. Plant Physiol. Plant Mol. Biol. 45, 369-392 (1994).

2. I. Andersson, Catalysis and regulation in Rubisco. J. Exp. Bot. 59, 1555-1568 (2008).

3. C. Bathellier, et al., Ribulose 1,5-bisphosphate carboxylase/oxygenase activates $\mathrm{O} 2$ by electron transfer. Proc Natl Acad Sci USA 117, 24234-24242 (2020).

4. A. I. Flamholz, et al., Revisiting Trade-offs between Rubisco Kinetic Parameters. Biochemistry 58, 3365-3376 (2019).

5. A. Flamholz, P. M. Shih, Cell biology of photosynthesis over geologic time. Curr. Biol. 30, R490-R494 (2020).

6. C. A. Kerfeld, M. R. Melnicki, Assembly, function and evolution of cyanobacterial carboxysomes. Curr. Opin. Plant Biol. 31, 66-75 (2016).

7. B. D. Rae, B. M. Long, M. R. Badger, G. D. Price, Functions, compositions, and evolution of the two types of carboxysomes: polyhedral microcompartments that facilitate $\mathrm{CO} 2$ fixation in cyanobacteria and some proteobacteria. Microbiol. Mol. Biol. Rev. 77, 357-379 (2013).

8. J. J. Desmarais, et al., DABs are inorganic carbon pumps found throughout prokaryotic phyla. Nat. Microbiol. 4, 2204-2215 (2019).

9. A. I. Flamholz, et al., Functional reconstitution of a bacterial $\mathrm{CO} 2$ concentrating mechanism in Escherichia coli. eLife 9 (2020).

10. F. Cai, et al., The pentameric vertex proteins are necessary for the icosahedral carboxysome shell to function as a CO2 leakage barrier. PLoS ONE 4, e7521 (2009).

11. N. Mangan, M. Brenner, Systems analysis of the $\mathrm{CO} 2$ concentrating mechanism in cyanobacteria. eLife, e02043 (2014).

12. N. M. Mangan, A. Flamholz, R. D. Hood, R. Milo, D. F. Savage, pH determines the energetic efficiency of the cyanobacterial $\mathrm{CO} 2$ concentrating mechanism. Proc Natl Acad Sci USA 113, E5354-62 (2016).

13. M. R. Melnicki, M. Sutter, C. A. Kerfeld, Evolutionary relationships among shell proteins of carboxysomes and metabolosomes. Curr. Opin. Microbiol. 63, 1-9 (2021).

14. M. S. Kimber, Carboxysomal carbonic anhydrases. Subcell Biochem 75, 89-103 (2014).

15. Z. Dou, et al., CO2 fixation kinetics of Halothiobacillus neapolitanus mutant carboxysomes lacking carbonic anhydrase suggest the shell acts as a diffusional barrier for CO2. J. Biol. Chem. 283, 10377-10384 (2008).

16. H. Fukuzawa, E. Suzuki, Y. Komukai, S. Miyachi, A gene homologous to chloroplast 
carbonic anhydrase (icfA) is essential to photosynthetic carbon dioxide fixation by Synechococcus PCC7942. Proc Natl Acad Sci USA 89, 4437-4441 (1992).

17. G. D. Price, M. R. Badger, Expression of Human Carbonic Anhydrase in the Cyanobacterium Synechococcus PCC7942 Creates a High CO(2)-Requiring Phenotype: Evidence for a Central Role for Carboxysomes in the $\mathrm{CO}(2)$ Concentrating Mechanism. Plant Physiol. 91, 505-513 (1989).

18. S. H. Baker, D. S. Williams, H. C. Aldrich, A. C. Gambrell, J. M. Shively, Identification and localization of the carboxysome peptide Csos3 and its corresponding gene in Thiobacillus neapolitanus. Arch. Microbiol. 173, 278-283 (2000).

19. A. K.-C. So, et al., A novel evolutionary lineage of carbonic anhydrase (epsilon class) is a component of the carboxysome shell. J. Bacteriol. 186, 623-630 (2004).

20. M. R. Sawaya, et al., The structure of beta-carbonic anhydrase from the carboxysomal shell reveals a distinct subclass with one active site for the price of two. J. Biol. Chem. 281, 7546-7555 (2006).

21. S. Heinhorst, et al., Characterization of the carboxysomal carbonic anhydrase CsoSCA from Halothiobacillus neapolitanus. J. Bacteriol. 188, 8087-8094 (2006).

22. K. L. Peña, S. E. Castel, C. de Araujo, G. S. Espie, M. S. Kimber, Structural basis of the oxidative activation of the carboxysomal gamma-carbonic anhydrase, CcmM. Proc Natl Acad Sci USA 107, 2455-2460 (2010).

23. C. de Araujo, et al., Identification and characterization of a carboxysomal y-carbonic anhydrase from the cyanobacterium Nostoc sp. PCC 7120. Photosyn. Res. 121, 135-150 (2014).

24. L. D. McGurn, et al., The structure, kinetics and interactions of the $\beta$-carboxysomal $\beta$-carbonic anhydrase, CcaA. Biochem. J. 473, 4559-4572 (2016).

25. B. M. Long, M. R. Badger, S. M. Whitney, G. D. Price, Analysis of carboxysomes from Synechococcus PCC7942 reveals multiple Rubisco complexes with carboxysomal proteins CcmM and CcaA. J. Biol. Chem. 282, 29323-29335 (2007).

26. L. M. Oltrogge, et al., Multivalent interactions between CsoS2 and Rubisco mediate a-carboxysome formation. Nat. Struct. Mol. Biol. 27, 281-287 (2020).

27. L. M. Oltrogge, et al., a-carboxysome formation is mediated by the multivalent and disordered protein CsoS2. BioRxiv (2019) https:/doi.org/10.1101/708164.

28. S. He, et al., The structural basis of Rubisco phase separation in the pyrenoid. Nat. Plants 6, 1480-1490 (2020).

29. M. T. Meyer, et al., Assembly of the algal CO2-fixing organelle, the pyrenoid, is guided by a Rubisco-binding motif. Sci. Adv. 6 (2020).

30. M. Wu, G. C. Lander, M. A. Herzik, Sub-2 Angstrom resolution structure determination using single-particle cryo-EM at $200 \mathrm{keV}$. Journal of Structural Biology: X 4, 100020 (2020).

31. F. Cai, et al., Advances in understanding carboxysome assembly in prochlorococcus and synechococcus implicate csos2 as a critical component. Life (Basel) 5, 1141-1171 (2015).

32. T. Chaijarasphong, et al., Programmed Ribosomal Frameshifting Mediates Expression of the a-Carboxysome. J. Mol. Biol. 428, 153-164 (2016).

33. S. Heinhorst, G. C. Cannon, J. M. Shively, "Carboxysomes and Carboxysome-like Inclusions" in Complex Intracellular Structures in Prokaryotes, Microbiology Monographs., J. M. Shively, Ed. (Springer Berlin Heidelberg, 2006), pp. 141-165.

34. F. Cai, S. Heinhorst, J. M. Shively, G. C. Cannon, Transcript analysis of the Halothiobacillus neapolitanus cso operon. Arch. Microbiol. 189, 141-150 (2008).

35. V. N. Uversky, Intrinsically disordered proteins in overcrowded milieu: Membrane-less 
organelles, phase separation, and intrinsic disorder. Curr. Opin. Struct. Biol. 44, 18-30 (2017).

36. Y.-C. C. Tsai, M. C. Lapina, S. Bhushan, O. Mueller-Cajar, Identification and characterization of multiple rubisco activases in chemoautotrophic bacteria. Nat. Commun. 6, 8883 (2015).

37. M. Sutter, et al., Structural Characterization of a Newly Identified Component of a-Carboxysomes: The AAA+ Domain Protein CsoCbbQ. Sci. Rep. 5, 16243 (2015).

38. $\mathrm{H}$. Wang, et al., Rubisco condensate formation by $\mathrm{CcmM}$ in $\beta$-carboxysome biogenesis. Nature 566, 131-135 (2019).

39. P. Ryan, et al., The small RbcS-like domains of the $\beta$-carboxysome structural protein $\mathrm{CcmM}$ bind RubisCO at a site distinct from that binding the RbcS subunit. J. Biol. Chem. 294, 2593-2603 (2019).

40. S. Lechno-Yossef, et al., Cyanobacterial carboxysomes contain an unique rubisco-activase-like protein. New Phytol. 225, 793-806 (2020).

41. M. Flecken, et al., Dual functions of a rubisco activase in metabolic repair and recruitment to carboxysomes. Cell 183, 457-473.e20 (2020).

42. L. C. M. Mackinder, et al., A repeat protein links Rubisco to form the eukaryotic carbon-concentrating organelle. Proc Natl Acad Sci USA 113, 5958-5963 (2016).

43. T. Wunder, S. L. H. Cheng, S.-K. Lai, H.-Y. Li, O. Mueller-Cajar, The phase separation underlying the pyrenoid-based microalgal Rubisco supercharger. Nat. Commun. 9, 5076 (2018).

44. V. M. Markowitz, et al., IMG: the Integrated Microbial Genomes database and comparative analysis system. Nucleic Acids Res. 40, D115-22 (2012).

45. F. Madeira, et al., The EMBL-EBI search and sequence analysis tools APIs in 2019. Nucleic Acids Res. 47, W636-W641 (2019).

46. A. M. Waterhouse, J. B. Procter, D. M. A. Martin, M. Clamp, G. J. Barton, Jalview Version 2--a multiple sequence alignment editor and analysis workbench. Bioinformatics 25, 1189-1191 (2009).

47. J. Trifinopoulos, L.-T. Nguyen, A. von Haeseler, B. Q. Minh, W-IQ-TREE: a fast online phylogenetic tool for maximum likelihood analysis. Nucleic Acids Res. 44, W232-5 (2016).

48. I. Letunic, P. Bork, Interactive Tree Of Life (iTOL) v5: an online tool for phylogenetic tree display and annotation. Nucleic Acids Res. 49, W293-W296 (2021).

49. D. T. Jones, D. Cozzetto, DISOPRED3: precise disordered region predictions with annotated protein-binding activity. Bioinformatics 31, 857-863 (2015).

50. T. L. Bailey, J. Johnson, C. E. Grant, W. S. Noble, The MEME Suite. Nucleic Acids Res. 43, W39-49 (2015).

51. A. Drozdetskiy, C. Cole, J. Procter, G. J. Barton, JPred4: a protein secondary structure prediction server. Nucleic Acids Res. 43, W389-94 (2015).

52. A. R. Hagen, et al., In vitro assembly of diverse bacterial microcompartment shell architectures. Nano Lett. 18, 7030-7037 (2018).

53. A. Punjani, J. L. Rubinstein, D. J. Fleet, M. A. Brubaker, cryoSPARC: algorithms for rapid unsupervised cryo-EM structure determination. Nat. Methods 14, 290-296 (2017).

54. J. Zivanov, et al., New tools for automated high-resolution cryo-EM structure determination in RELION-3. eLife 7 (2018).

55. A. Rohou, N. Grigorieff, CTFFIND4: Fast and accurate defocus estimation from electron micrographs. J. Struct. Biol. 192, 216-221 (2015).

56. A. Casañal, B. Lohkamp, P. Emsley, Current developments in Coot for macromolecular model building of Electron Cryo-microscopy and Crystallographic Data. Protein Sci. 29, 
1069-1078 (2020).

57. D. Liebschner, et al., Macromolecular structure determination using X-rays, neutrons and electrons: recent developments in Phenix. Acta Crystallogr. D Struct. Biol. 75, 861-877 (2019).

58. T. C. Terwilliger, S. J. Ludtke, R. J. Read, P. D. Adams, P. V. Afonine, Improvement of cryo-EM maps by density modification. Nat. Methods 17, 923-927 (2020).

59. T. C. Terwilliger, O. V. Sobolev, P. V. Afonine, P. D. Adams, Automated map sharpening by maximization of detail and connectivity. Acta Crystallogr. D Struct. Biol. 74, 545-559 (2018).

60. P. V. Afonine, et al., Real-space refinement in PHENIX for cryo-EM and crystallography. Acta Crystallogr. D Struct. Biol. 74, 531-544 (2018).

61. T. D. Goddard, et al., UCSF ChimeraX: Meeting modern challenges in visualization and analysis. Protein Sci. 27, 14-25 (2018).

62. E. Krissinel, K. Henrick, Inference of macromolecular assemblies from crystalline state. J. Mol. Biol. 372, 774-797 (2007). 


\section{Discovery of a carbonic anhydrase-Rubisco supercomplex within the alpha-carboxysome}

Blikstad et al., 2021

Supplemental Information 
bioRxiv preprint doi: https://doi.org/10.1101/2021.11.05.467472. this version posted November 5,2021 . The copyright holder for this preprint (which was not certified by peer review) is the author/funder, who has granted bioRxiv a license to display the preprint in perpetuity. It is made available under aCC-BY 4.0 International license.

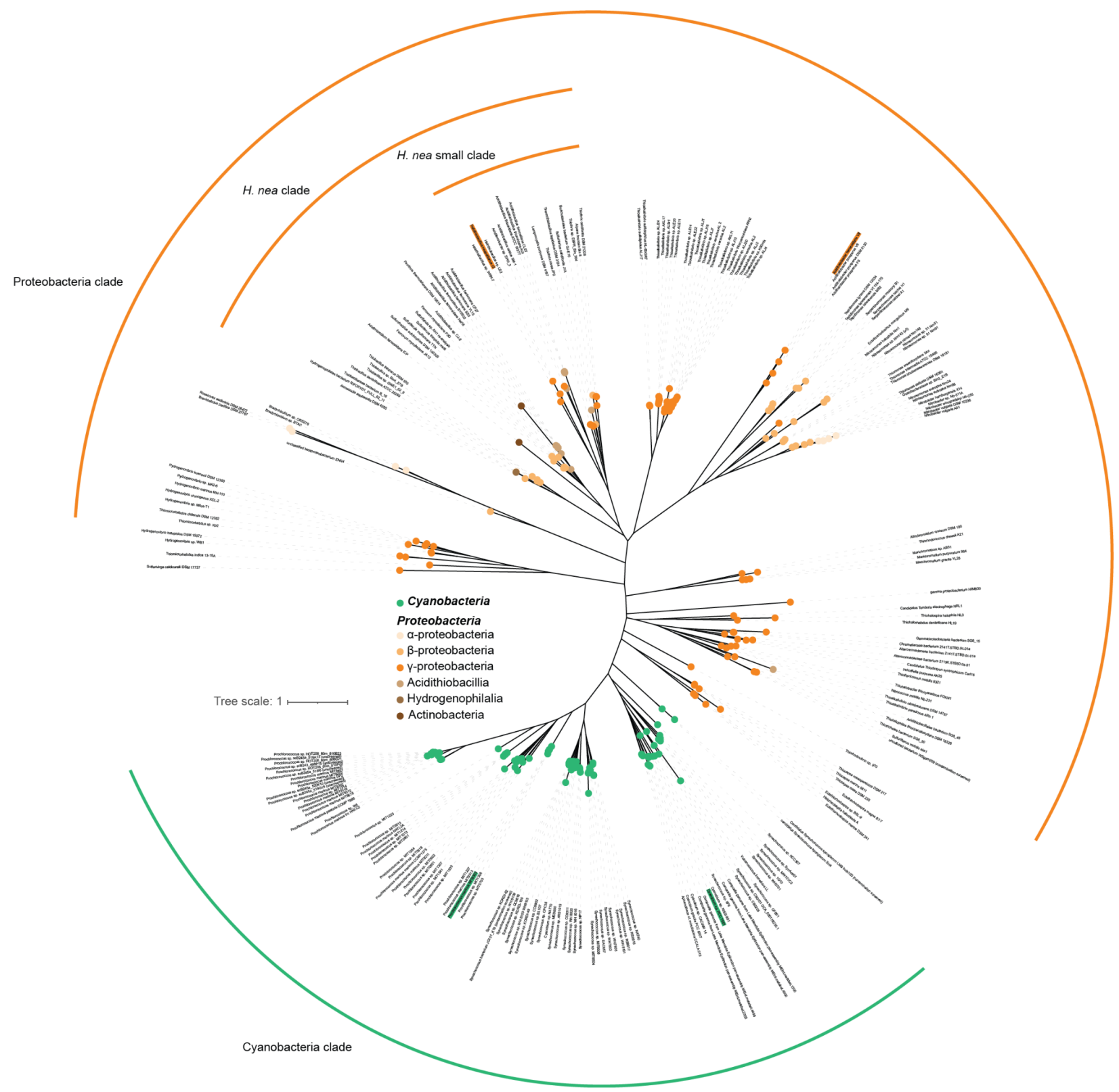

SI Figure 1: Phylogenetic tree of CsoSCA

Annotated maximum-likelihood phylogenetic tree of CsoSCA. Cyanobacterial homologs are colored in green and proteobacteria homologous in an orange/brown gradient. Scale bar, 0.1 substitutions per site. 
bioRxiv preprint doi: https://doi.org/10.1101/2021.11.05.467472; this version posted November 5, 2021. The copyright holder for this preprint (which was not certified by peer review) is the author/funder, who has granted bioRxiv a license to display the preprint in perpetuity. It is made available under aCC-BY 4.0 International license.
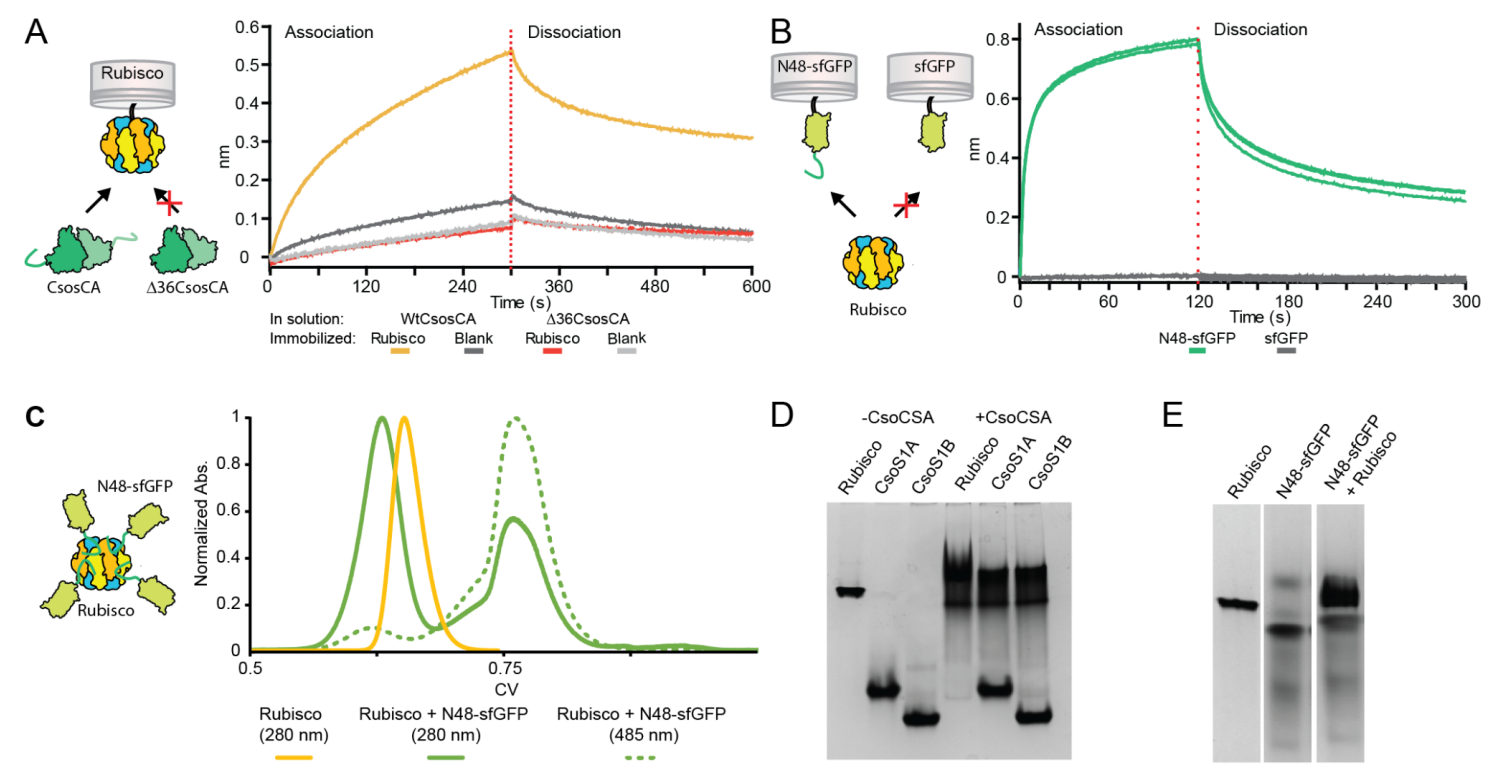

SI Figure 2: CsoSCA-Rubisco interaction controls

(A) BLI response showing that full length CsoSCA binds to Rubisco but a NTD truncated version ( $\triangle 36 \mathrm{CsoSCA})$ does not bind. (B) BLI response showing that Rubisco binds to N48-sfGFP but not to the negative control sfGFP. (C) Size exclusion chromatography experiment showing co-elution of Rubisco and N48-sfGFP, demonstrating the interaction in a solution based assay. (D) Native-page demonstrating binding between Rubisco and CsoSCA, and lack of binding between the major shell proteins CsoS1A and CsoS1B. (E) Native-page demonstrating interaction between Rubisco and N48-sfGFP. 
bioRxiv preprint doi: https://doi.org/10.1101/2021.11.05.467472. this version posted November 5,2021 . The copyright holder for this preprint (which was not certified by peer review) is the author/funder, who has granted bioRxiv a license to display the preprint in perpetuity. It is made available under aCC-BY 4.0 International license.

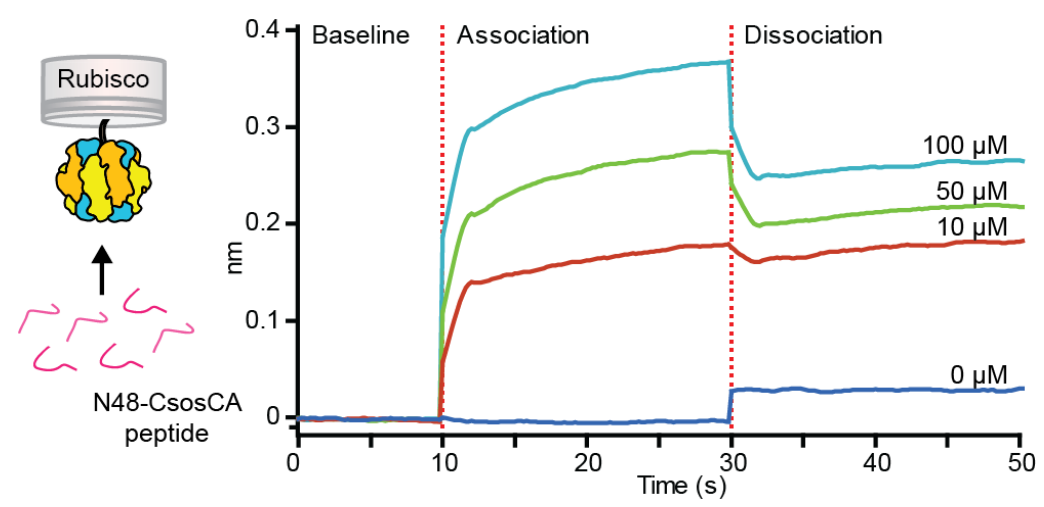

SI Figure 3: Binding of N48-peptide to immobilized Rubisco

$\mathrm{BLI}$ response showing binding of the N48-peptide to Rubisco. Binding was measured with three peptide concentrations (10,50 and $100 \mu \mathrm{M}$ peptide). 
bioRxiv preprint doi: https://doi.org/10.1101/2021.11.05.467472; this version posted November 5, 2021. The copyright holder for this preprint (which was not certified by peer review) is the author/funder, who has granted bioRxiv a license to display the preprint in perpetuity. It is made available under aCC-BY 4.0 International license.
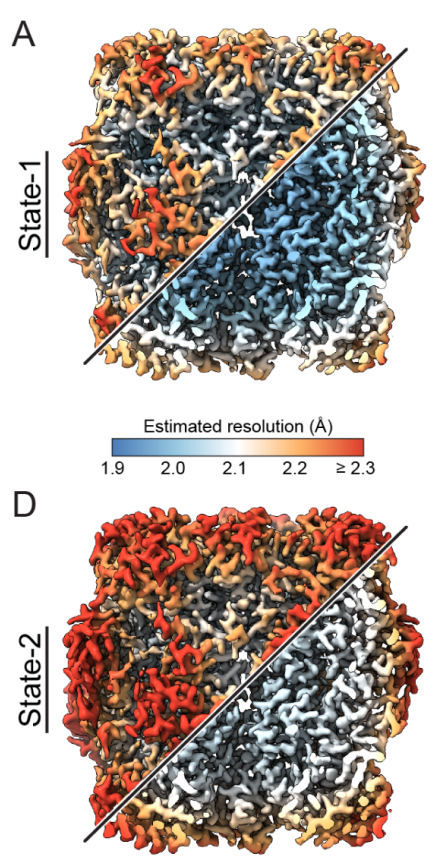

B

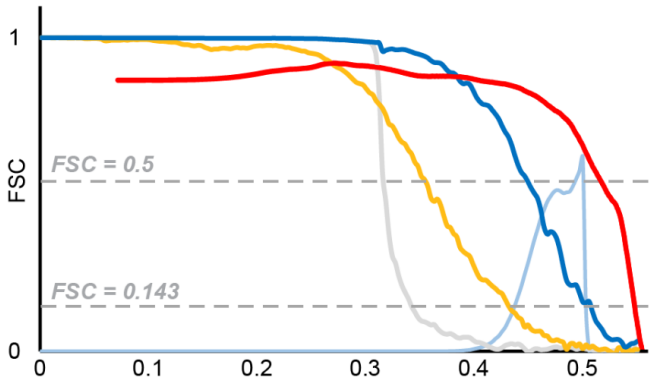

OPhase-rand. OUnmasked $\bigcirc$ Masked O Local res. OModel vs. map

$\mathrm{E}$

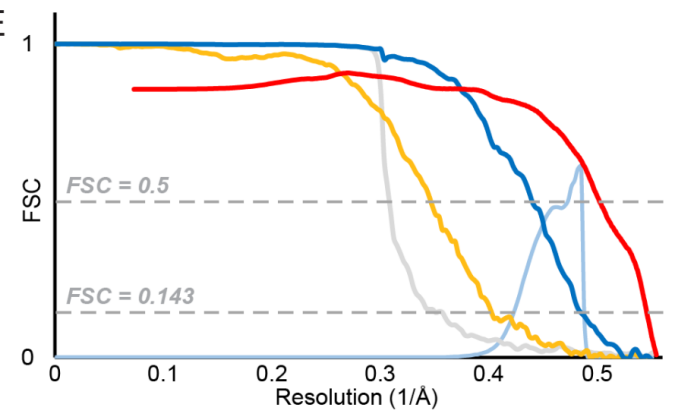

C

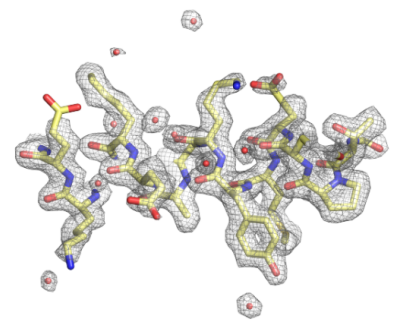

CbbL: $237-255$

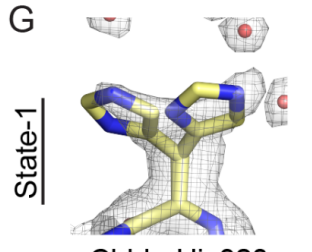

CbbL: His320

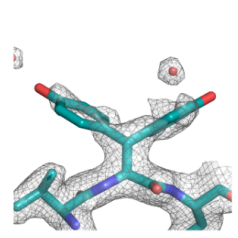

CbbS: Tyr107

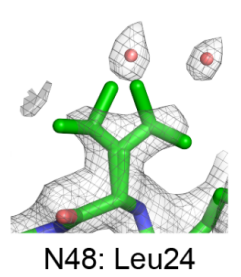

$\mathrm{H}$

$\mathrm{F}$
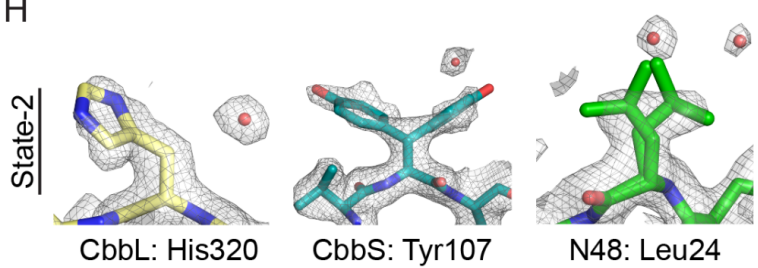

(6)

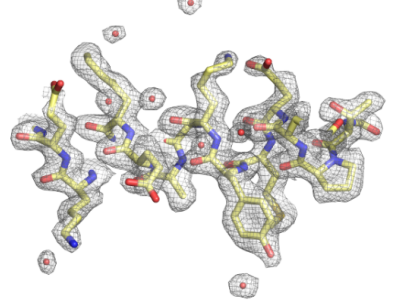

SI Figure 4: Resolution estimates and visual quality of Rubisco-N48 cryo-EM reconstructions

(A) Local resolution estimate of State-1 map. The map is shown in unsharpened and locally filtered by estimated resolution. (B) Half-map and map-model FSC curves for State-1 map. (C) Exemplar State-1 density of an internal helix of $\mathrm{CbbL}$ from the density-modified and sharpened map contoured at $2 \sigma$. (D-F) Same as A-C for the State-2 cryo-EM reconstruction. (G,H) Model and density for residues with resolved alternate rotamer confirmations in either State-1 or State-2 reconstructions. Density shown is from the respective density-modified and sharpened map contoured at $2 \sigma$. 


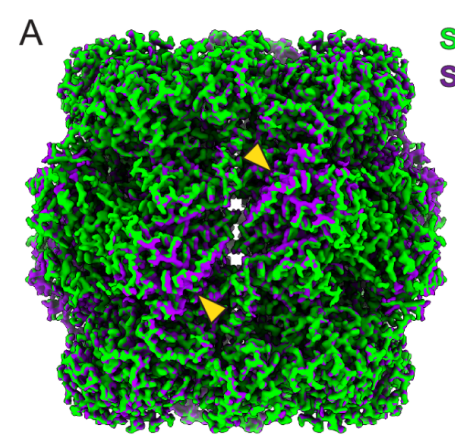

C

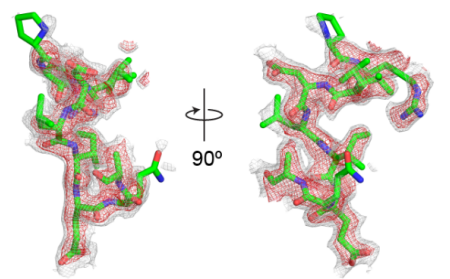

State $=1$

State-2

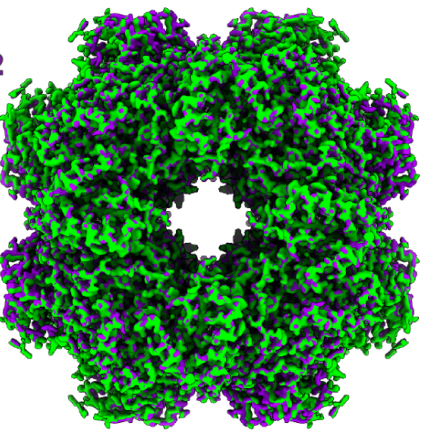

D

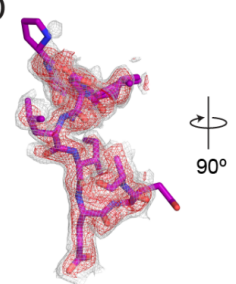

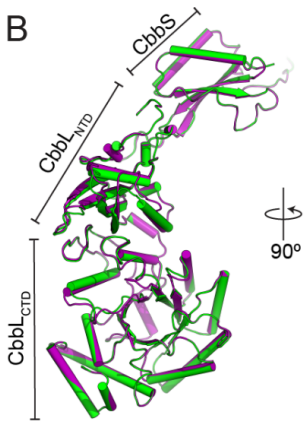

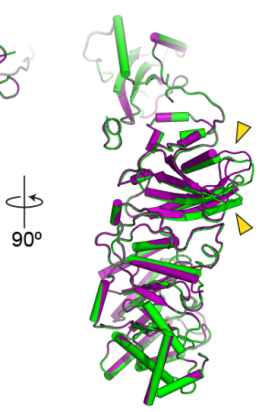

E

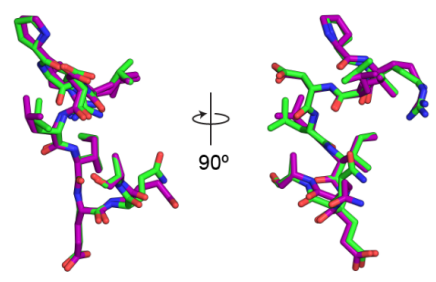

SI Figure 5: Comparison of the two reconstructed Rubisco-N48 states

(A) Overlay of the two cryo-EM maps with State-1 in green and State-2 in purple. Yellow arrows point to regions of noticeable difference. (B) Asymmetric units of coordinate models for the two states, colored the same as in A. Subunits and domains are labelled and yellow arrows point to differences in loop confirmation in the $\mathrm{CbbL}_{\text {NTD. }}$ (C) State-1 N48-peptide coordinate model and density contoured at $1.5 \sigma$ (grey) and $2 \sigma$ (red) from the density-modified, sharpened map. (D) Same as in C for State-2. (E) Overlay of N48 coordinate models of the two states. 


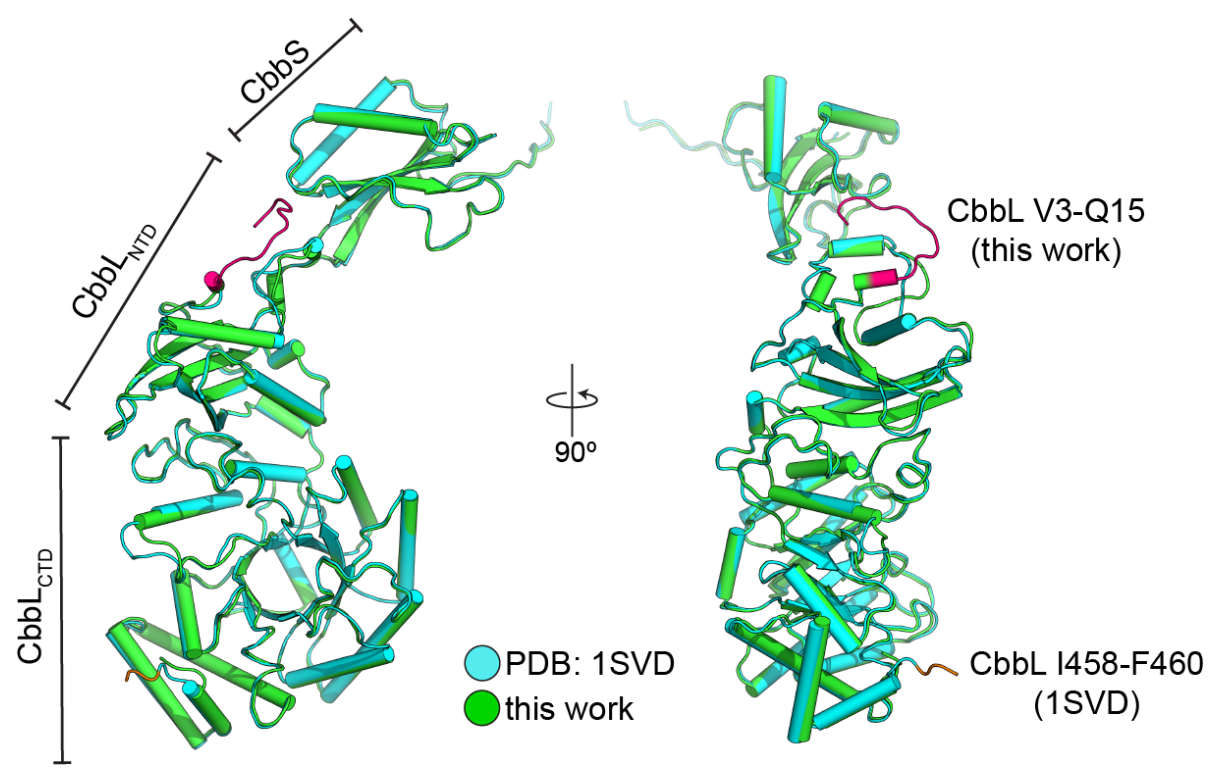

SI Figure 6: Comparison of cryo-EM and crystal structures of Hnea CbbL/S

Overlay of CbbL/S from State-1 (this work) in green and the previous X-ray crystal structure (PDB:1svd) in cyan. Subunits and domain distincts are labelled. Difference in the extent of resolved termini of CbbL between these structures are highlighted. Magenta coloring indicates additional N-terminal CbbL residues resolved in this work. Orange coloring indicates $\mathrm{C}$-terminal $\mathrm{CbbL}$ residues resolved in the crystal structure. 
bioRxiv preprint doi: https://doi.org/10.1101/2021.11.05.467472; this version posted November 5, 2021. The copyright holder for this preprint (which was not certified by peer review) is the author/funder, who has granted bioRxiv a license to display the preprint in perpetuity. It is made available under aCC-BY 4.0 International license.

A

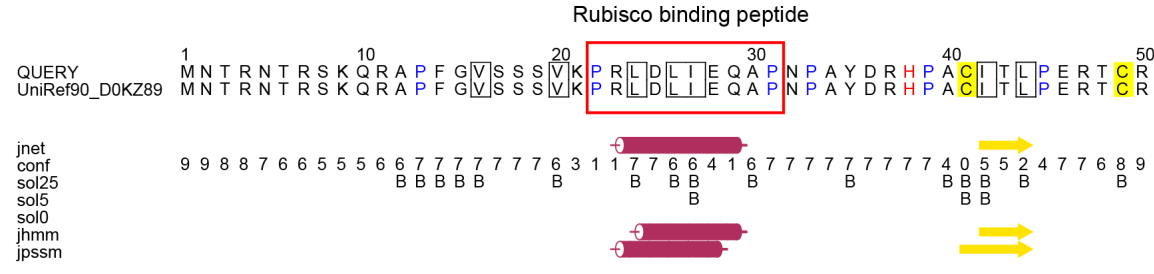

B

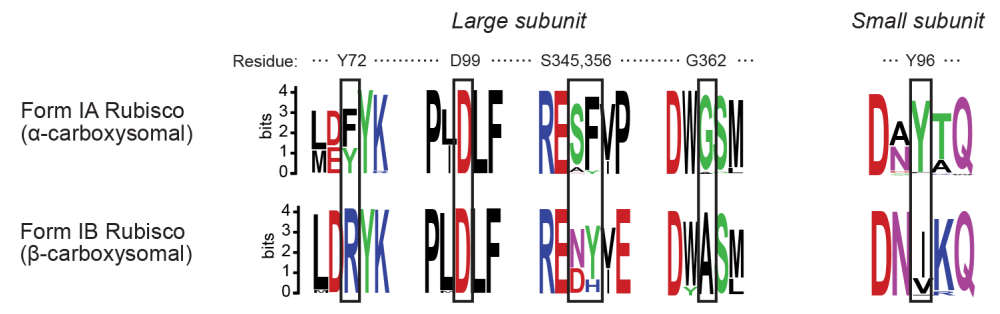

SI Figure 7: Secondary structure prediction of N48-peptid and Rubisco sequence comparison

(A) JPred4 protein secondary structure prediction server was used to predict the secondary structure of CsoSCA's N48 sequence. The PRLDLIEQAP sequence is predicted to form an alpha-helical structure. This stretch of sequence corresponds to the extra density observed in the cryoEM structure of Rubisco in complex with the N48 peptide (red box). (B) Rubisco sequence comparison at the CsoSCA-peptide interaction site. Multiple sequence alignment of $\alpha$-carboxysomal Form IA Rubiscos and $\beta$-carboxysomal Form IB Rubiscos visualised using Weblogo. Residues are numbered according to the Rubisco $H$. neapolitanus sequence. Residues shown in our Rubisco $H$. neapolitanus structure to interact with the CsoSCA peptide are marked with a black box. CsoSCA interacting residues have a high conservation score but are in general not conserved between $\alpha$-carboxysomal and $\beta$-carboxysomal Rubiscos. 

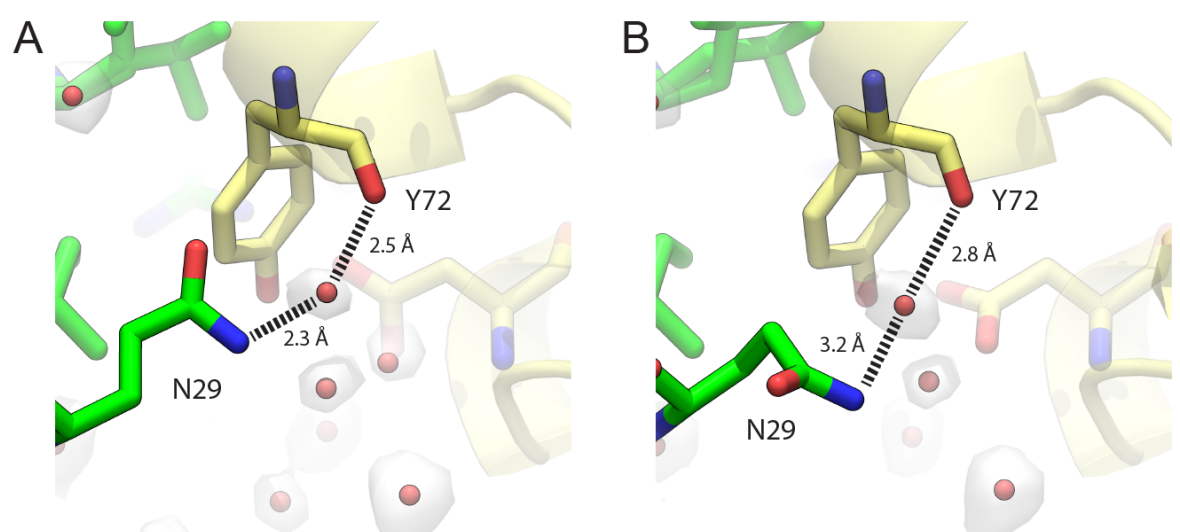

$\underline{\text { State-1 }}$
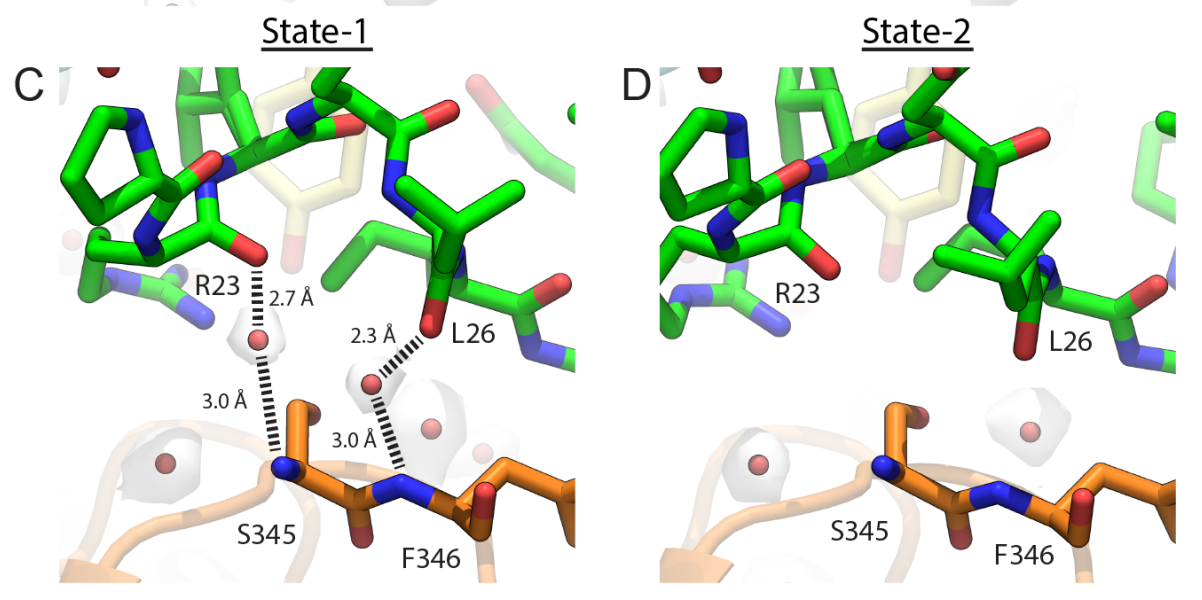

SI Figure 8: Putative ordered waters mediating interaction between N48-peptide and Rubisco

$(A, B)$ Water-mediated interaction between N48-peptide and $\mathrm{CbbL}_{\mathrm{A}}$ resolved in both Rubisco-N48 maps. (C,D) Water-mediated interactions between N48-peptide and $\mathrm{CbbL}_{B}$ resolved in the State-1 (C, $1.98 \AA$ ) map but not in State-2 (D, $2.07 \AA)$ map. Putative ordered water densities are shown as transparent white surfaces contoured at $1.5 \sigma$ from respective density-modified and sharpened maps. 
bioRxiv preprint doi: https://doi. org/10.1101/2021.11.05.467472. this version posted November 5,2021 . The copyright holder for this preprint (which was not certified by peer review) is the author/funder, who has granted bioRxiv a license to display the preprint in perpetuity. It is made available under aCC-BY 4.0 International license.

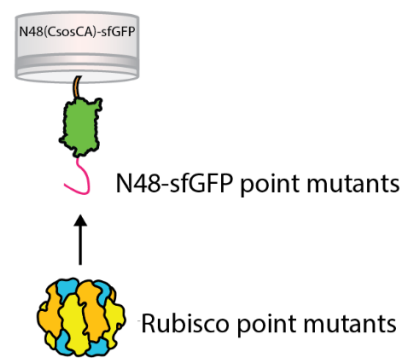

P22A N48-sfGFP

vs. 3300 - $52 \mathrm{nM}$ Rubisco
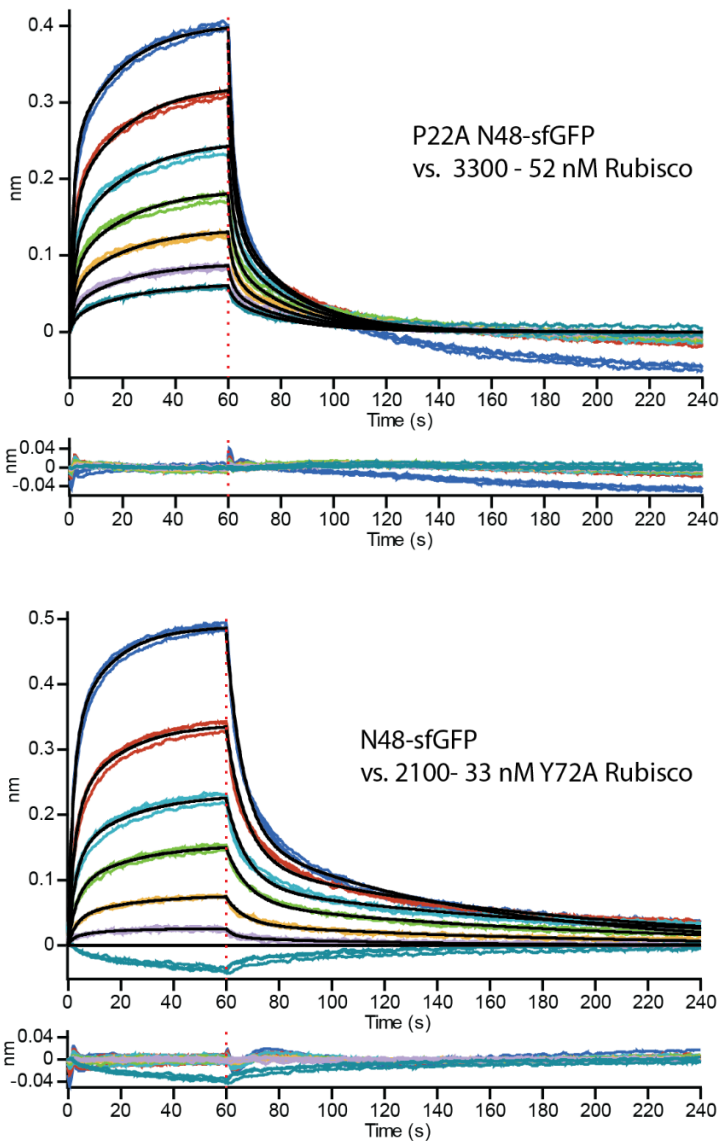
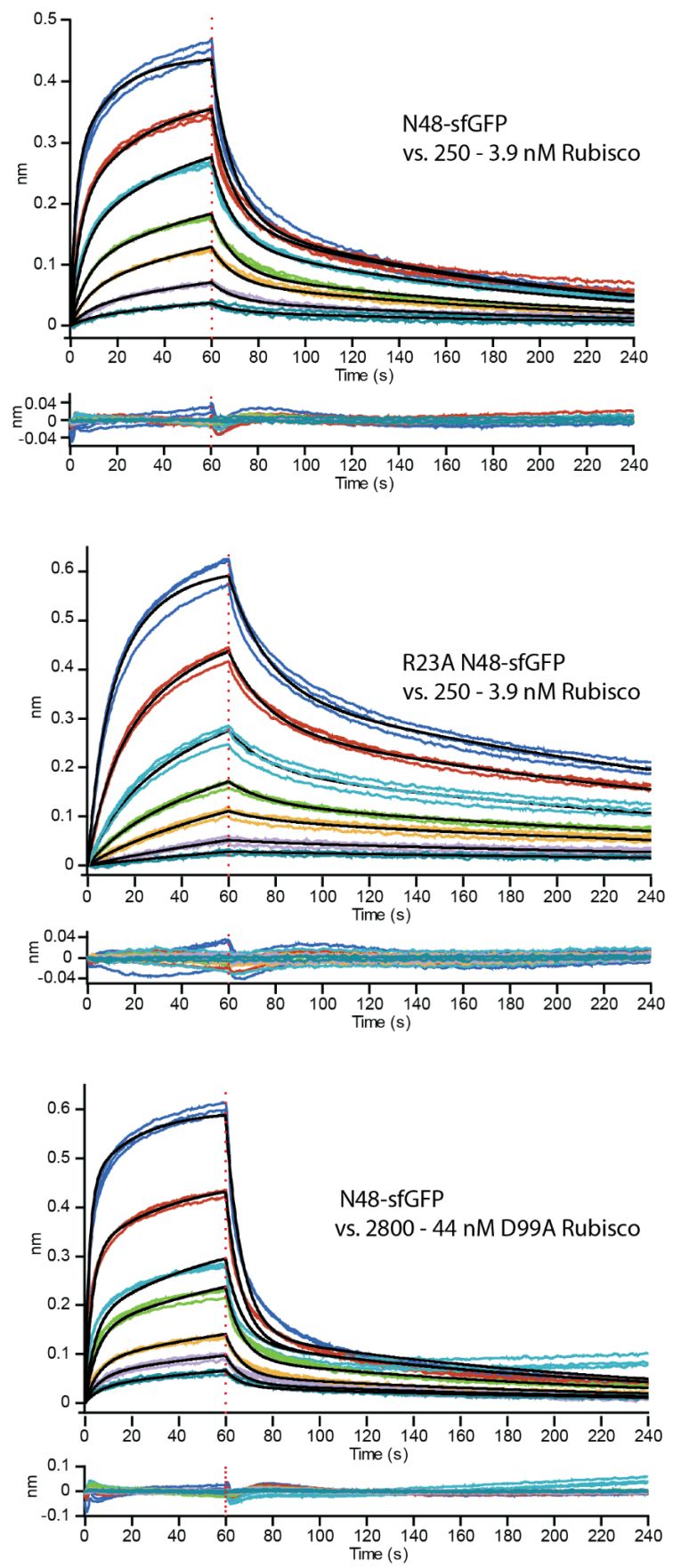

SI Flgure 9: BLI sensograms of N48-sfGFP and Rubisco point mutants

BLI response from binding affinity measurements of Rubisco against immobilized N48-sfGFP using point mutants of Rubisco or of N48-sfGFP. Mutant and concentration range of Rubisco are indicated in figure. $K_{\mathrm{D}}, k_{\mathrm{on}}$ and $k_{\text {off }}$ are listed in Table 1. 
bioRxiv preprint doi: https://doi.org/10.1101/2021.11.05.467472; this version posted November 5, 2021. The copyright holder for this preprint (which was not certified by peer review) is the author/funder, who has granted bioRxiv a license to display the preprint in perpetuity. It is made available under aCC-BY 4.0 International license.

A
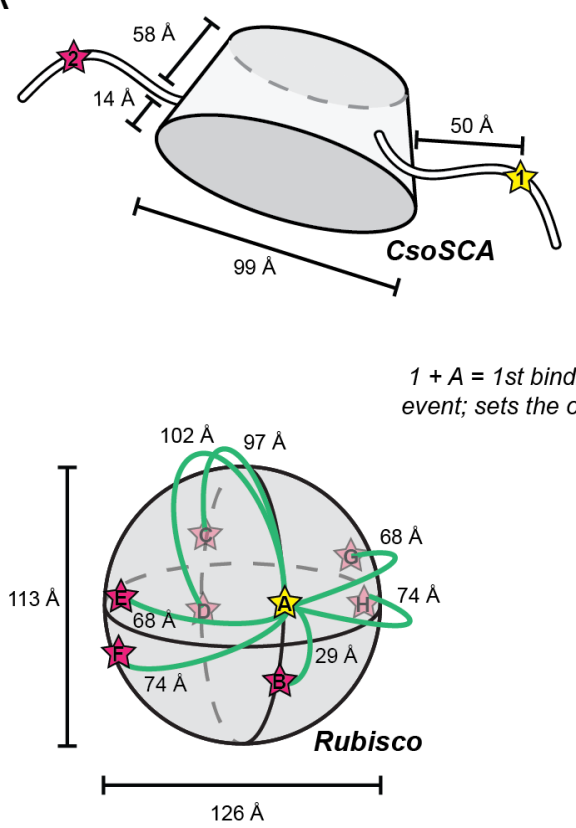

B Examples of possible

CsoscA-Rubisco interaction modes

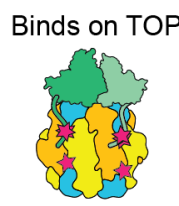

Binds on SIDE

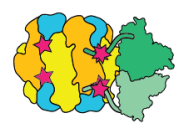

Binds TWO Rubisco's

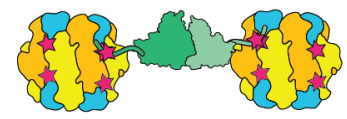

SI Figure 10: Possible binding conformations of CsoSCA-Rubisco.

(A) Diagram showing the approximate distances between binding motif on CsoSCA (top) and binding sites on Rubisco (bottom). Binding motifs/sites are marked with a star. (B) Cartoon representation of three examples of possible CsoSCA-Rubisco interaction modes. 
bioRxiv preprint doi: https://doi.org/10.1101/2021.11.05.467472; this version posted November 5, 2021. The copyright holder for this preprint (which was not certified by peer review) is the author/funder, who has granted bioRxiv a license to display the preprint in perpetuity. It is made available under aCC-BY 4.0 International license.

A
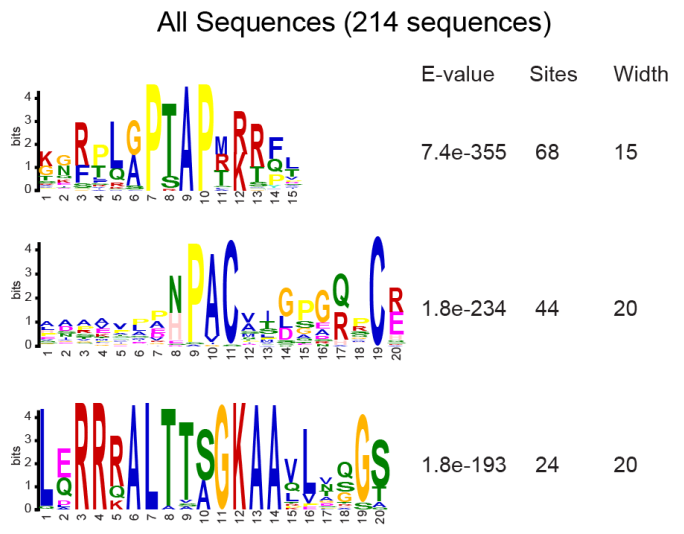

Cyanobacteria clade (85 sequences)
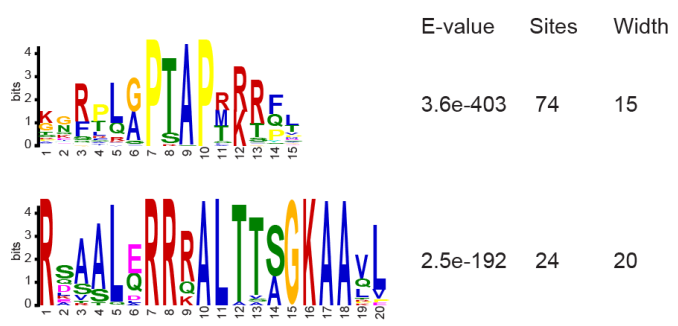

$2.5 \mathrm{e}-192 \quad 24 \quad 20$

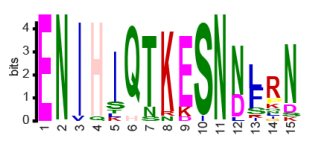

$5.2 \mathrm{e}-114$
Proteobacteria clade (129 sequences)
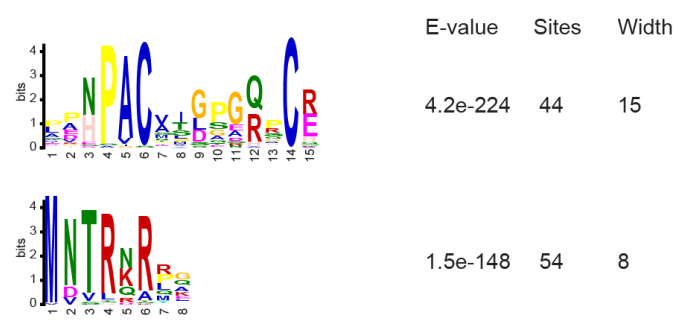

$1.5 \mathrm{e}-148 \quad 54 \quad 8$

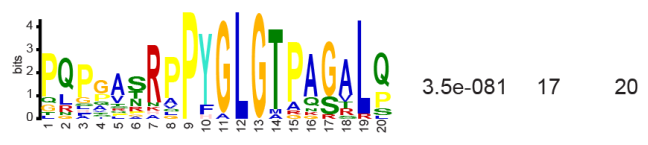

H. neapolitanus clade (37 sequences)

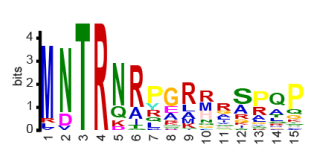

E-value Sites Width

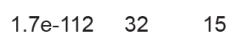
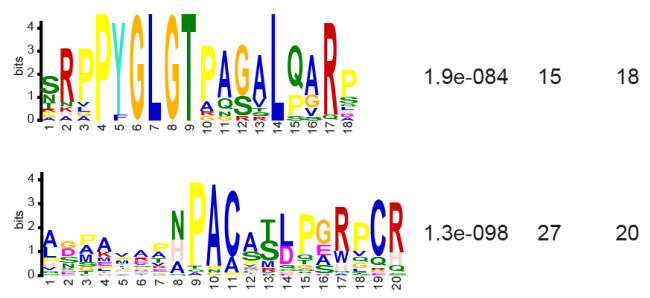

B

Halothiobacillus neapolitanus
Halothiobacillus sp. LS2
Halothiobacillus sp. WRN-7

Thiothrix caldifontis DSM 21228

Burkholderiales bacterium GJ-E10

Thermithiobacillus tepidarius DSM 3134

\begin{abstract}
$V \stackrel{1}{1}^{2}$ R L D L I E Q A P P P A
T Q P R L D A E T V Q P P

GA P R L G R R A P F G G

H T P R K T H P T P V A L

VVPRTAQP $\mathrm{P}$ G V A A A

P S P R P VA T S A Q P A
\end{abstract}

SI Figure 11: Conservation analysis of CsoSCA NTD Rubisco binding motif

(A) Motif discovery of CsoSCAs NTD sequence using MEME analysis (Multiple Em for Motif Elicitation). Analysis was performed on all CsoSCA sequences, as well as sequences specific to the Proteobacteria and Cyanobacteria (SI Table 1). For a more stringent analysis, the $H$. neapolitanus clade was analysed separately as well. 44/129 of the collected Proteobacteria sequences contain a PACxxxxxxxC motif. H.neapolitanus CsoSCA has this motif, however, it does not appear to be essential for binding to Rubisco. The N-terminus MNTRxR is somewhat conserved in Proteobacteria (55/129 sequences). In Cyanobacterial CsoSCAs a conserved PTAPxRR motif (75/85 sequences) is identified. 24/85 Cyanobacteria sequences contain what appears to be the conserved CsoS2 Rubisco binding motif, RxxxxxRRRxxxxxGK. These sequences all belong to the Procloroccocos genus. The experimentally identified $H$. neapolitanus CsoSCA Rubisco binding motif, PRLDLIEQA, does not appear to be conserved among homologos. (B) Alignment of a selection of sequences from $H$. neapolitanus CsoSCAs closest homologs. Bold letters indicate residues conserved with the $H$. neapolitanus motif. Its closest homologue Halothiobacillus sp. LS2 contains the same motif. Some of the others have the PR residues, demonstrated to be essential for binding, however the rest of the motif is poorly conserved. 